\title{
SOME OPERATOR BOUNDS EMPLOYING COMPLEX INTERPOLATION REVISITED
}

\author{
FRITZ GESZTESY, YURI LATUSHKIN, FEDOR SUKOCHEV, AND YURI TOMILOV
}

Dedicated with great pleasure to Charles Batty on the occasion of his 60th birthday.

\begin{abstract}
We revisit and extend known bounds on operator-valued functions of the type

$$
T_{1}^{-z} S T_{2}^{-1+z}, \quad z \in \bar{\Sigma}=\{z \in \mathbb{C} \mid \operatorname{Re}(z) \in[0,1]\},
$$

under various hypotheses on the linear operators $S$ and $T_{j}, j=1,2$. We particularly single out the case of self-adjoint and sectorial operators $T_{j}$ in some separable complex Hilbert space $\mathcal{H}_{j}, j=1,2$, and suppose that $S$ (resp., $S^{*}$ ) is a densely defined closed operator mapping $\operatorname{dom}(S) \subseteq \mathcal{H}_{1}$ into $\mathcal{H}_{2}$ (resp., $\operatorname{dom}\left(S^{*}\right) \subseteq \mathcal{H}_{2}$ into $\mathcal{H}_{1}$ ), relatively bounded with respect to $T_{1}$ (resp., $T_{2}^{*}$ ). Using complex interpolation methods, a generalized polar decomposition for $S$, and Heinz's inequality, the bounds we establish lead to inequalities of the following type: Given $k \in(0, \infty)$,
\end{abstract}

$$
\begin{aligned}
& \left\|\overline{T_{2}^{-z} S T_{1}^{-1+z}}\right\|_{\mathcal{B}\left(\mathcal{H}_{1}, \mathcal{H}_{2}\right)} \\
& \leq N_{1} N_{2} e^{k(\operatorname{Im}(z))^{2}+k \operatorname{Re}(z)[1-\operatorname{Re}(z)]+(4 k)^{-1}\left(\theta_{1}+\theta_{2}\right)^{2}} \\
& \times\left\|S T_{1}^{-1}\right\|_{\mathcal{B}\left(\mathcal{H}_{1}, \mathcal{H}_{2}\right)}^{1-\operatorname{Re}(z)}\left\|S^{*}\left(T_{2}^{*}\right)^{-1}\right\|_{\mathcal{B}\left(\mathcal{H}_{2}, \mathcal{H}_{1}\right)}^{\operatorname{Re}(z)}, \quad z \in \bar{\Sigma},
\end{aligned}
$$

which also implies,

$$
\begin{aligned}
& \left\|\overline{T_{2}^{-x} S T_{1}^{-1+x}}\right\|_{\mathcal{B}\left(\mathcal{H}_{1}, \mathcal{H}_{2}\right)} \leq N_{1} N_{2} e^{\left(\theta_{1}+\theta_{2}\right)[x(1-x)]^{1 / 2}} \\
& \quad \times\left\|S T_{1}^{-1}\right\|_{\mathcal{B}\left(\mathcal{H}_{1}, \mathcal{H}_{2}\right)}^{1-x}\left\|S^{*}\left(T_{2}^{*}\right)^{-1}\right\|_{\mathcal{B}\left(\mathcal{H}_{2}, \mathcal{H}_{1}\right)}^{x}, \quad x \in[0,1],
\end{aligned}
$$

assuming that $T_{j}$ have bounded imaginary powers, that is, for some $N_{j} \geqslant 1$ and $\theta_{j} \geqslant 0$,

$$
\left\|T_{j}^{i s}\right\|_{\mathcal{B}(\mathcal{H})} \leq N_{j} e^{\theta_{j}|s|}, \quad s \in \mathbb{R}, j=1,2 .
$$

We also derive analogous bounds with $\mathcal{B}\left(\mathcal{H}_{1}, \mathcal{H}_{2}\right)$ replaced by trace ideals, $\mathcal{B}_{p}\left(\mathcal{H}_{1}, \mathcal{H}_{2}\right), p \in[1, \infty)$. The methods employed are elementary, predominantly relying on Hadamard's three-lines theorem and Heinz's inequality.

\section{INTRODUCTION}

This paper was inspired by an interesting result proved by Lesch in Appendix A to his 2005 paper [22, dealing with uniqueness of spectral flow on spaces of unbounded Fredholm operators. More precisely, upon a close inspection of the proof

Date: June 8, 2018.

2010 Mathematics Subject Classification. Primary 47A57, 47B10, 47B44; Secondary 47A30, $47 \mathrm{~B} 25$.

Key words and phrases. Complex interpolation, generalized Heinz-type inequalities, operator and trace norm inequalities.

Partially supported by the NSF grant DMS-1067929, by the Research Board and the Research Council of the University of Missouri, by the ARC, the NCN grant DEC-2011/03/B/ST1/00407, and by the EU Marie Curie IRSES program, project "AOS", No. 318910. 
of [22, Proposition A.1], we derived the following interpolation result in [6] (cf. 6. Theorem 4.1]):

Theorem 1.1. Let $\mathcal{H}$ be a separable Hilbert space and $T \geq 0$ be a self-adjoint operator with $T^{-1} \in \mathcal{B}(\mathcal{H})$. Assume that $S$ is closed and densely defined in $\mathcal{H}$, with $\left(\operatorname{dom}(S) \cap \operatorname{dom}\left(S^{*}\right)\right) \supseteq \operatorname{dom}(T)$, implying $S T^{-1} \in \mathcal{B}(\mathcal{H})$ and $S^{*} T^{-1} \in \mathcal{B}(\mathcal{H})$. If, in addition, $S T^{-1} \in \mathcal{B}_{1}(\mathcal{H})$ and $S^{*} T^{-1} \in \mathcal{B}_{1}(\mathcal{H})$, then

$$
T^{-1 / 2} S T^{-1 / 2} \in \mathcal{B}_{1}(\mathcal{H}), \quad\left(T^{-1 / 2} S T^{-1 / 2}\right)^{*}=T^{-1 / 2} S^{*} T^{-1 / 2} \in \mathcal{B}_{1}(\mathcal{H}) .
$$

Moreover,

$$
\left\|T^{-1 / 2} S T^{-1 / 2}\right\|_{\mathcal{B}_{1}(\mathcal{H})}=\left\|T^{-1 / 2} S^{*} T^{-1 / 2}\right\|_{\mathcal{B}_{1}(\mathcal{H})} \leqslant\left\|S T^{-1}\right\|_{\mathcal{B}_{1}(\mathcal{H})}^{1 / 2}\left\|S^{*} T^{-1}\right\|_{\mathcal{B}_{1}(\mathcal{H})}^{1 / 2} .
$$

Theorem 1.1 was used repeatedly in 6] (in Section 4 and especially, in Section 6). We then announced the present paper in 2010, but due to a variety of reasons, finishing it was delayed for quite a while. We should also mention that in the meantime we became aware of a paper by Huang [14], who proved, in fact, extended, some parts of Lesch's Proposition A.1 in 22] already in 1988 (we will return to this in Sections 2 and 3 ).

Given Theorem 1.1, we became interested in extensions of it of the following three types:

- The case of fractional powers of $T$ different from $1 / 2$.

- General trace ideals $\mathcal{B}_{p}(\mathcal{H}), p \in(1, \infty)$.

- Classes of non-self-adjoint operators $T$, especially, sectorial operators $T$ having bounded imaginary powers.

While interpolation theory has long been raised to a high art, we emphasize that the methods we use are entirely elementary, being grounded in complex interpolation, particularly, in Hadamard's three-lines theorem as pioneered by Kato [18, Sect. 3], and Heinz's inequality. In fact, Kato [18, Sect. 3] presents a proof of the generalized Heinz inequality applying Hadamard's three-lines theorem, and hence the latter is the ultimate ingredient in our proofs.

We continue with a brief summary of the content of each section. One of the principal results proven in Section 2 reads as follows: Assume that $T_{j}$ are selfadjoint operators in $\mathcal{H}_{j}$ with $T_{j}^{-1} \in \mathcal{B}\left(\mathcal{H}_{j}\right), j=1,2$, and suppose that $S$ is a closed operator mapping $\operatorname{dom}(S) \subseteq \mathcal{H}_{1}$ into $\mathcal{H}_{2}$ satisfying $\operatorname{dom}(S) \supseteq \operatorname{dom}\left(T_{1}\right)$ and $\operatorname{dom}\left(S^{*}\right) \supseteq \operatorname{dom}\left(T_{2}\right)$. Then $T_{2}^{-z} S T_{1}^{-1+z}$ defined on $\operatorname{dom}\left(T_{1}\right), z \in \bar{\Sigma}$, is closable, and given $k \in(0, \infty)$, one obtains

$$
\begin{aligned}
& \left\|\overline{T_{2}^{-z} S T_{1}^{-1+z}}\right\|_{\mathcal{B}\left(\mathcal{H}_{1}, \mathcal{H}_{2}\right)} \leq\left\|S T_{1}^{-1}\right\|_{\mathcal{B}\left(\mathcal{H}_{1}, \mathcal{H}_{2}\right)}^{1-\operatorname{Re}(z)}\left\|S^{*} T_{2}^{-1}\right\|_{\mathcal{B}\left(\mathcal{H}_{2}, \mathcal{H}_{1}\right)}^{\operatorname{Re}(z)} \\
& \quad \times\left\{\begin{array}{l}
e^{k|\operatorname{Im}(z)|^{2}+k \operatorname{Re}(z)[1-\operatorname{Re}(z)]+k^{-1} \pi^{2}}, \\
e^{k|\operatorname{Im}(z)|^{2}+k \operatorname{Re}(z)[1-\operatorname{Re}(z)]+(4 k)^{-1} \pi^{2}}, \text { if } T_{1} \geq 0, \text { or } T_{2} \geq 0, \quad z \in \bar{\Sigma}, \\
1, \text { if } T_{j} \geq 0, j=1,2,
\end{array}\right.
\end{aligned}
$$

as well as

$$
\left\|\overline{T_{2}^{-x} S T_{1}^{-1+x}}\right\|_{\mathcal{B}\left(\mathcal{H}_{1}, \mathcal{H}_{2}\right)} \leq\left\|S T_{1}^{-1}\right\|_{\mathcal{B}\left(\mathcal{H}_{1}, \mathcal{H}_{2}\right)}^{1-x}\left\|S^{*} T_{2}^{-1}\right\|_{\mathcal{B}\left(\mathcal{H}_{2}, \mathcal{H}_{1}\right)}^{x}
$$




$$
\times\left\{\begin{array}{l}
e^{2 \pi[x(1-x)]^{1 / 2}}, \\
e^{\pi[x(1-x)]^{1 / 2}}, \text { if } T_{1} \geq 0, \text { or } T_{2} \geq 0, \\
1, \text { if } T_{j} \geq 0, j=1,2
\end{array} \quad x \in[0,1]\right.
$$

In Section 3 we turn to trace ideals $\mathcal{B}_{p}(\mathcal{H}), p \in(1, \infty)$. In addition to the hypotheses imposed on $T_{j}, j=1,2$, and $S$ mentioned in the paragraph preceding (1.3), let $p \in[1, \infty)$ and $S T_{1}^{-1} \in \mathcal{B}_{p}\left(\mathcal{H}_{1}, \mathcal{H}_{2}\right)$ and $S^{*} T_{2}^{-1} \in \mathcal{B}_{p}\left(\mathcal{H}_{2}, \mathcal{H}_{1}\right)$. Then given $k \in(0, \infty)$, the principal result in Section 3 derives the analog of (1.3) and (1.4) in the form,

$$
\begin{aligned}
& \left\|\overline{T_{2}^{-z} S T_{1}^{-1+z}}\right\|_{\mathcal{B}_{p}\left(\mathcal{H}_{1}, \mathcal{H}_{2}\right)} \leq\left\|S T_{1}^{-1}\right\|_{\mathcal{B}_{p}\left(\mathcal{H}_{1}, \mathcal{H}_{2}\right)}^{1-\operatorname{Re}(z)}\left\|S^{*} T_{2}^{-1}\right\|_{\mathcal{B}_{p}\left(\mathcal{H}_{2}, \mathcal{H}_{1}\right)}^{\operatorname{Re}(z)} \\
& \quad \times\left\{\begin{array}{l}
e^{k|\operatorname{Im}(z)|^{2}+k \operatorname{Re}(z)[1-\operatorname{Re}(z)]+k^{-1} \pi^{2}}, \\
e^{k|\operatorname{Im}(z)|^{2}+k \operatorname{Re}(z)[1-\operatorname{Re}(z)]+(4 k)^{-1} \pi^{2}}, \text { if } T_{1} \geq 0, \text { or } T_{2} \geq 0, \quad z \in \bar{\Sigma}, \\
1, \text { if } T_{j} \geq 0, j=1,2,
\end{array}\right.
\end{aligned}
$$

as well as

$$
\begin{aligned}
& \left\|\overline{T_{2}^{-x} S T_{1}^{-1+x}}\right\|_{\mathcal{B}_{p}\left(\mathcal{H}_{1}, \mathcal{H}_{2}\right)} \leq\left\|S T_{1}^{-1}\right\|_{\mathcal{B}_{p}\left(\mathcal{H}_{1}, \mathcal{H}_{2}\right)}^{1-x}\left\|S^{*} T_{2}^{-1}\right\|_{\mathcal{B}_{p}\left(\mathcal{H}_{2}, \mathcal{H}_{1}\right)}^{x} \\
& \quad \times\left\{\begin{array}{l}
e^{2 \pi[x(1-x)]^{1 / 2}}, \\
e^{\pi[x(1-x)]^{1 / 2}}, \text { if } T_{1} \geq 0, \text { or } T_{2} \geq 0, \\
1, \text { if } T_{j} \geq 0, j=1,2,
\end{array}\right.
\end{aligned}
$$

In our final Section 4 we discuss the extension of (1.3) and (1.4) from selfadjoint to sectorial operators $T_{j}, j=1,2$. One of our principal results there reads as follows: Assume that $T_{j}$ are sectorial operators in $\mathcal{H}_{j}$ such that $T_{j}^{-1} \in \mathcal{B}\left(\mathcal{H}_{j}\right)$, and that for some $\theta_{j} \geq 0, N_{j} \geq 1,\left\|T_{j}^{i s}\right\|_{\mathcal{B}\left(\mathcal{H}_{j}\right)} \leq N_{j} e^{\theta_{j}|s|}, s \in \mathbb{R}, j=1,2$. In addition, suppose that $S$ is a closed operator mapping $\operatorname{dom}(S) \subseteq \mathcal{H}_{1}$ into $\mathcal{H}_{2}$, satisfying $\operatorname{dom}(S) \supseteq \operatorname{dom}\left(T_{1}\right)$ and $\operatorname{dom}\left(S^{*}\right) \supseteq \operatorname{dom}\left(T_{2}^{*}\right)$. Then $T_{2}^{-z} S T_{1}^{-1+z}$ defined on $\operatorname{dom}\left(T_{1}\right), z \in \bar{\Sigma}$, is closable, and given $k \in(0, \infty)$, one obtains

$$
\begin{aligned}
& \left\|\overline{T_{2}^{-z} S T_{1}^{-1+z}}\right\|_{\mathcal{B}\left(\mathcal{H}_{1}, \mathcal{H}_{2}\right)} \leq N_{1} N_{2} e^{k(\operatorname{Im}(z))^{2}+k \operatorname{Re}(z)[1-\operatorname{Re}(z)]+(4 k)^{-1}\left(\theta_{1}+\theta_{2}\right)^{2}} \\
& \quad \times\left\|S T_{1}^{-1}\right\|_{\mathcal{B}\left(\mathcal{H}_{1}, \mathcal{H}_{2}\right)}^{1-\operatorname{Re}(z)}\left\|S^{*}\left(T_{2}^{*}\right)^{-1}\right\|_{\mathcal{B}\left(\mathcal{H}_{2}, \mathcal{H}_{1}\right)}^{\operatorname{Re}(z)}, \quad z \in \bar{\Sigma}
\end{aligned}
$$

as well as

$$
\begin{aligned}
& \left\|\overline{T_{2}^{-x} S T_{1}^{-1+x}}\right\|_{\mathcal{B}\left(\mathcal{H}_{1}, \mathcal{H}_{2}\right)} \leq N_{1} N_{2} e^{\left(\theta_{1}+\theta_{2}\right)[x(1-x)]^{1 / 2}} \\
& \quad \times\left\|S T_{1}^{-1}\right\|_{\mathcal{B}\left(\mathcal{H}_{1}, \mathcal{H}_{2}\right)}^{1-x}\left\|S^{*}\left(T_{2}^{*}\right)^{-1}\right\|_{\mathcal{B}\left(\mathcal{H}_{2}, \mathcal{H}_{1}\right)}^{x}, \quad x \in[0,1] .
\end{aligned}
$$

Moreover, in addition to the hypotheses on $T_{j}, j=1,2$, and $S$ mentioned in the paragraph preceding (1.7), let $p \in[1, \infty)$ and $S T_{1}^{-1} \in \mathcal{B}_{p}\left(\mathcal{H}_{1}, \mathcal{H}_{2}\right), S^{*}\left(T_{2}^{*}\right)^{-1} \in$ $\mathcal{B}_{p}\left(\mathcal{H}_{2}, \mathcal{H}_{1}\right)$. Then given $k \in(0, \infty)$, one obtains the analog of (1.5) and (1.6) in the form,

$$
\begin{aligned}
& \left\|\overline{T_{2}^{-z} S T_{1}^{-1+z}}\right\|_{\mathcal{B}_{p}\left(\mathcal{H}_{1}, \mathcal{H}_{2}\right)} \leq N_{1} N_{2} e^{k(\operatorname{Im}(z))^{2}+k \operatorname{Re}(z)[1-\operatorname{Re}(z)]+(4 k)^{-1}\left(\theta_{1}+\theta_{2}\right)^{2}} \\
& \quad \times\left\|S T_{1}^{-1}\right\|_{\mathcal{B}_{p}\left(\mathcal{H}_{1}, \mathcal{H}_{2}\right)}^{1-\operatorname{Re}(z)}\left\|S^{*}\left(T_{2}^{*}\right)^{-1}\right\|_{\mathcal{B}_{p}\left(\mathcal{H}_{2}, \mathcal{H}_{1}\right)}^{\operatorname{Re}(z)}, z \in \bar{\Sigma}
\end{aligned}
$$


as well as

$$
\begin{aligned}
& \left\|\overline{T_{2}^{-x} S T_{1}^{-1+x}}\right\|_{\mathcal{B}_{p}\left(\mathcal{H}_{1}, \mathcal{H}_{2}\right)} \leq N_{1} N_{2} e^{\left(\theta_{1}+\theta_{2}\right)[x(1-x)]^{1 / 2}} \\
& \quad \times\left\|S T_{1}^{-1}\right\|_{\mathcal{B}_{p}\left(\mathcal{H}_{1}, \mathcal{H}_{2}\right)}^{1-x}\left\|S^{*}\left(T_{2}^{*}\right)^{-1}\right\|_{\mathcal{B}_{p}\left(\mathcal{H}_{2}, \mathcal{H}_{1}\right)}^{x}, \quad x \in[0,1] .
\end{aligned}
$$

We note that we permit operators $T$ in (1.3) - (1.6) to have spectrum covering $\mathbb{R}$ except for a neighborhood of zero. Thus, our results in Section 4 for sectorial operators $T$ do not cover the results (1.3)-(1.6).

In conclusion, we briefly summarize the basic notation used in this paper: Let $\mathcal{H}$ be a separable complex Hilbert space, $(\cdot, \cdot)_{\mathcal{H}}$ the scalar product in $\mathcal{H}$ (linear in the second factor), and $I_{\mathcal{H}}$ the identity operator in $\mathcal{H}$. Limits in the norm topology on $\mathcal{H}$ (also called strong limits in $\mathcal{H}$ ) will be denoted by s-lim. If $T$ is a linear operator mapping (a subspace of) a Hilbert space into another, $\operatorname{dom}(T)$ denotes the domain of $T$. The closure of a closable operator $S$ is denoted by $\bar{S}$. The spectrum and resolvent set of a closed linear operator in $\mathcal{H}$ will be denoted by $\sigma(\cdot)$ and $\rho(\cdot)$, respectively. The Banach spaces of bounded and compact linear operators in $\mathcal{H}$ are denoted by $\mathcal{B}(\mathcal{H})$ and $\mathcal{B}_{\infty}(\mathcal{H})$, respectively; in the context of two Hilbert spaces, $\mathcal{H}_{j}, j=1,2$, we use the analogous abbreviations $\mathcal{B}\left(\mathcal{H}_{1}, \mathcal{H}_{2}\right)$ and $\mathcal{B}_{\infty}\left(\mathcal{H}_{1}, \mathcal{H}_{2}\right)$. Similarly, the usual $\ell^{p}$-based Schatten-von Neumann (trace) ideals are denoted by $\mathcal{B}_{p}(\mathcal{H}), p \in[1, \infty)$.

\section{Interpolation and some Operator Norm Bounds Revisited}

In this section we revisit and extend a number of bounds collected by Lesch in [22, Proposition A.1]:

Through most of this section we will make the following assumptions:

Hypothesis 2.1. Assume that $T$ is a self-adjoint operator in $\mathcal{H}$ with $T^{-1} \in \mathcal{B}(\mathcal{H})$. In addition, suppose that $S$ is a closed operator in $\mathcal{H}$ satisfying

$$
\operatorname{dom}(S) \cap \operatorname{dom}\left(S^{*}\right) \supseteq \operatorname{dom}(T) .
$$

In particular, Hypothesis 2.1 implies that

$$
S T^{-1} \in \mathcal{B}(\mathcal{H}), \quad S^{*} T^{-1} \in \mathcal{B}(\mathcal{H}) .
$$

Remark 2.2. ( $i)$ In the sequel we will adhere to the following convention: Operator products $A B$ of two linear operators $A$ and $B$ in $\mathcal{H}$ are always assumed to be maximally defined, that is,

$$
\operatorname{dom}(A B)=\{f \in \mathcal{H} \mid f \in \operatorname{dom}(B), B f \in \operatorname{dom}(A)\},
$$

unless explicitly stated otherwise. The same convention is of course applied to products of three or more linear operators in $\mathcal{H}$.

(ii) We recall the following useful facts (see, e.g., [41, Theorem 4.19 ]): Suppose $T_{j}, j=1,2$, are two densely defined linear operators in $\mathcal{H}$ such that $T_{2} T_{1}$ is also densely defined in $\mathcal{H}$. Then,

$$
\left(T_{2} T_{1}\right)^{*} \supseteq T_{1}^{*} T_{2}^{*}
$$

If in addition $T_{2} \in \mathcal{B}(\mathcal{H})$, then

$$
\left(T_{2} T_{1}\right)^{*}=T_{1}^{*} T_{2}^{*}
$$


Theorem 2.3. Assume Hypothesis 2.1, Then the following facts hold:

(i) The operators $T^{-1} S T$ and $T^{-1} S^{*} T$ are well-defined on $\operatorname{dom}\left(T^{2}\right)$, and hence densely defined in $\mathcal{H}$,

$$
\operatorname{dom}\left(T^{-1} S T\right) \cap \operatorname{dom}\left(T^{-1} S^{*} T\right) \supseteq \operatorname{dom}\left(T^{2}\right) .
$$

(ii) The relations

$$
\left(T^{-1} S T\right)^{*}=T S^{*} T^{-1}, \quad\left(T^{-1} S^{*} T\right)^{*}=T S T^{-1},
$$

hold, and hence $T S^{*} T^{-1}$ and $T S T^{-1}$ are closed in $\mathcal{H}$.

(iii) One infers that

$$
T^{-1} S T \text { is bounded if and only if }\left(T^{-1} S T\right)^{*}=T S^{*} T^{-1} \in \mathcal{B}(\mathcal{H}) .
$$

In case $T^{-1} S T$ is bounded, then

$$
\overline{T^{-1} S T}=\left(T S^{*} T^{-1}\right)^{*}, \quad\left\|\overline{T^{-1} S T}\right\|_{\mathcal{B}(\mathcal{H})}=\left\|T S^{*} T^{-1}\right\|_{\mathcal{B}(\mathcal{H})} .
$$

Analogously, one concludes that

$$
T^{-1} S^{*} T \text { is bounded if and only if }\left(T^{-1} S^{*} T\right)^{*}=T S T^{-1} \in \mathcal{B}(\mathcal{H}) \text {. }
$$

In case $T^{-1} S^{*} T$ is bounded, then

$$
\overline{T^{-1} S^{*} T}=\left(T S T^{-1}\right)^{*}, \quad\left\|\overline{T^{-1} S^{*} T}\right\|_{\mathcal{B}(\mathcal{H})}=\left\|T S T^{-1}\right\|_{\mathcal{B}(\mathcal{H})} .
$$

Proof. We start by recalling that

$$
\begin{aligned}
\operatorname{dom}\left(T^{-1} S T\right) & =\{g \in \operatorname{dom}(T) \mid T g \in \operatorname{dom}(S)\}, \\
\operatorname{dom}\left(T S^{*} T^{-1}\right) & =\left\{f \in \mathcal{H} \mid S^{*} T^{-1} f \in \operatorname{dom}(T)\right\} .
\end{aligned}
$$

(i) Suppose that $g \in \operatorname{dom}\left(T^{2}\right)$. Then $T g \in \operatorname{dom}(T) \subseteq \operatorname{dom}(S)$ and hence

$$
\operatorname{dom}\left(T^{-1} S T\right) \supseteq \operatorname{dom}\left(T^{2}\right) .
$$

Since $T$ and hence $T^{2}$ are self-adjoint in $\mathcal{H}$ and hence necessarily densely defined, $T^{-1} S T$ is densely defined in $\mathcal{H}$. The same applies to $T^{-1} S^{*} T$.

(ii) Applying Remark 2.2 (ii), one obtains

$$
\left(T^{-1} S T\right)^{*}=\left(T^{-1}[S T]\right)^{*}=[S T]^{*} T^{-1} \supseteq T S^{*} T^{-1},
$$

since $T^{-1} S T$ is densely defined in $\mathcal{H}$ by item $(i)$. To prove the converse inclusion in (2.15), we now assume that $f \in \operatorname{dom}\left(\left(T^{-1} S T\right)^{*}\right)$ and $g \in \operatorname{dom}\left(T^{2}\right) \subseteq$ $\operatorname{dom}\left(T^{-1} S T\right)$. Then

$$
\left(\left(T^{-1} S T\right)^{*} f, g\right)_{\mathcal{H}}=\left(f, T^{-1} S T g\right)_{\mathcal{H}}=\left(T^{-1} f, S T g\right)_{\mathcal{H}}=\left(S^{*} T^{-1} f, T g\right)_{\mathcal{H}} .
$$

Since $\operatorname{dom}\left(T^{2}\right)$ is an operator core for $T$, (2.16) extends to all $g \in \operatorname{dom}(T)$, that is, one has

$$
\left(\left(T^{-1} S T\right)^{*} f, g\right)_{\mathcal{H}}=\left(S^{*} T^{-1} f, T g\right)_{\mathcal{H}}, \quad f \in \operatorname{dom}\left(\left(T^{-1} S T\right)^{*}\right), g \in \operatorname{dom}(T) .
$$

Consequently, $S^{*} T^{-1} f \in \operatorname{dom}(T)$ and

$$
\begin{array}{r}
\left(\left(T^{-1} S T\right)^{*} f, g\right)_{\mathcal{H}}=\left(S^{*} T^{-1} f, T g\right)_{\mathcal{H}}=\left(T S^{*} T^{-1} f, g\right)_{\mathcal{H}}, \\
f \in \operatorname{dom}\left(\left(T^{-1} S T\right)^{*}\right), g \in \operatorname{dom}(T),
\end{array}
$$

implying

$$
\left(T^{-1} S T\right)^{*} f=T S^{*} T^{-1} f, \quad f \in \operatorname{dom}\left(\left(T^{-1} S T\right)^{*}\right),
$$


and hence,

$$
\left(T^{-1} S T\right)^{*} \subseteq T S^{*} T^{-1}
$$

Then (2.15) and (2.20) yield the first relation in (2.7). Replacing $S$ by $S^{*}$ yields the second relation in (2.7).

(iii) Since $T^{-1} S T$ is densely defined by (2.6), an application of [41, Theorem 4.14(a)] yields (2.8). Equation (2.9) is an immediate consequence of (2.8).

Again, replacing $S$ by $S^{*}$ implies (2.10) and (2.11).

In the following we denote by $\Sigma \subset \mathbb{C}$ the open strip

$$
\Sigma=\{z \in \mathbb{C} \mid \operatorname{Re}(z) \in(0,1)\}
$$

and by $\bar{\Sigma}$ its closure.

To state additional results we will have to apply a version of Hadamard's threelines theorem and hence recall the following general result:

Theorem 2.4 ([13] (see also [8], Sect. III.13)). Suppose $\phi(\cdot)$ is an analytic function on $\Sigma$, continuous on $\bar{\Sigma}$, and satisfying for some fixed $C \in \mathbb{R}$ and $a \in[0, \pi)$,

$$
\sup _{z \in \bar{\Sigma}}\left[e^{-a|\operatorname{Im}(z)|} \ln (|\phi(z)|)\right] \leq C \text {. }
$$

Then

$$
\begin{array}{r}
|\phi(z)| \leq \exp \left\{\frac { \operatorname { s i n } ( \pi \operatorname { R e } ( z ) ) } { 2 } \int _ { \mathbb { R } } d y \left[\frac{\ln (\phi(i y))}{\cosh (\pi(y-\operatorname{Im}(z)))-\cosh (\pi \operatorname{Re}(z))}\right.\right. \\
\left.\left.+\frac{\ln (\phi(1+i y))}{\cosh (\pi(y-\operatorname{Im}(z)))+\cosh (\pi \operatorname{Re}(z))}\right]\right\}, \\
z \in \Sigma .
\end{array}
$$

If in addition, for some $C_{0}, C_{1} \in(0, \infty)$,

$$
|\phi(i y)| \leq C_{0}, \quad|\phi(1+i y)| \leq C_{1}, \quad y \in \mathbb{R},
$$

then

$$
|\phi(z)| \leq C_{0}^{1-\operatorname{Re}(z)} C_{1}^{\operatorname{Re}(z)}, \quad z \in \bar{\Sigma}
$$

For a recent detailed exposition of such results we refer to [10, Sects. 1.3.2, 1.3.3]. A classical application of Theorem 2.4 to linear operators appeared in 34] (see also [3. Sect. 4.3]).

The growth condition (2.22) is of course familiar from Phragmen-Lindelöf-type arguments applied to the strip $\Sigma$ (see, e.g., [31, Theorem 12.9]).

In the sequel, complex powers $T^{z}, z \in \bar{\Sigma}$, of a self-adjoint operator $T$ in $\mathcal{H}$, with $T^{-1} \in \mathcal{B}(\mathcal{H})$, are defined in terms of the spectral representation of $T$,

$$
T=\int_{\sigma(T)} \lambda d E_{T}(\lambda),
$$

with $\left\{E_{T}(\lambda)\right\}_{\lambda \in \mathbb{R}}$ denoting the family of spectral projections of $T$, as follows: Since by hypothesis, $(-\varepsilon, \varepsilon) \cap \sigma(T)=\emptyset$ for some $\varepsilon>0$, one defines

$$
T^{z}=\int_{\sigma(T)} \lambda^{z} d E_{T}(\lambda), \quad z \in \bar{\Sigma},
$$

where

$$
\lambda^{z}=\lambda^{\operatorname{Re}(z)} e^{i \operatorname{Im}(z) \ln (|\lambda|)}\left[\theta(\lambda)+e^{-\pi \operatorname{Im}(z)} \theta(-\lambda)\right], \quad \lambda \in \mathbb{R} \backslash\{0\}, z \in \bar{\Sigma},
$$


and

$$
\theta(x)= \begin{cases}1, & x>0 \\ 0, & x<0\end{cases}
$$

Consequently, one obtains the estimate

$$
\left\|T^{i y}\right\|_{\mathcal{B}(\mathcal{H})} \leq \max \left(1, e^{-\pi y}\right) \leq e^{\pi|y|}, \quad y \in \mathbb{R}
$$

and

if $T \geq \varepsilon I_{\mathcal{H}}$, for some $\varepsilon>0$, then $T^{i y}$ is unitary, $\left\|T^{i y}\right\|_{\mathcal{B}(\mathcal{H})}=1, \quad y \in \mathbb{R}$.

Theorem 2.5. Assume Hypothesis 2.1 and suppose that $T S T^{-1} \in \mathcal{B}(\mathcal{H})$ as well as $T S^{*} T^{-1} \in \mathcal{B}(\mathcal{H})$. Then $S \in \mathcal{B}(\mathcal{H})$ (and hence $S^{*} \in \mathcal{B}(\mathcal{H})$ ) and

$$
\|S\|_{\mathcal{B}(\mathcal{H})}=\left\|S^{*}\right\|_{\mathcal{B}(\mathcal{H})} \leq\left\|T S T^{-1}\right\|_{\mathcal{B}(\mathcal{H})}^{1 / 2}\left\|T S^{*} T^{-1}\right\|_{\mathcal{B}(\mathcal{H})}^{1 / 2}\left\{\begin{array}{l}
e^{2 \pi} \\
1, \text { if } T \geq 0
\end{array}\right.
$$

Proof. Introducing

$\varphi_{k}(z)=e^{k z(z-1)}\left(T^{2} f, T^{2 z-3} S T^{-1-2 z} T^{2} g\right)_{\mathcal{H}}, \quad f, g \in \operatorname{dom}\left(T^{2}\right), z \in \bar{\Sigma}, k \in[0, \infty)$,

one infers that $\varphi_{k}$ is analytic on $\Sigma$. (We note that the idea to exploit the factor $e^{k z(z-1)}, k>0$, can already be found in the proof of [18, Theorem 6]. This factor is used in (2.34) - (2.36) below to neutralize factors of the type $e^{4 \pi|y|}$ and $e^{4 \pi|\operatorname{Im}(z)|}$.)

In the following we focus on the general case where $T$ is self-adjoint and $k>0$; in this case we will employ the bound (2.30).

Assuming $k>0$, 2.30) yields the estimates

$$
\begin{aligned}
\left|\varphi_{k}(i y)\right| & =e^{-k y^{2}}\left|\left(f, T^{2 i y-1} S T^{1-2 i y} g\right)_{\mathcal{H}}\right|=e^{-k y^{2}}\left|\left(T^{-2 i y} f,\left[T^{-1} S T\right] T^{-2 i y} g\right)_{\mathcal{H}}\right| \\
& \leq e^{-k y^{2}+4 \pi|y|}\left\|\overline{T^{-1} S T}\right\|_{\mathcal{B}(\mathcal{H})}\|f\|_{\mathcal{H}}\|g\|_{\mathcal{H}} \\
& =e^{-k y^{2}+4 \pi|y|}\left\|T S^{*} T^{-1}\right\|_{\mathcal{B}(\mathcal{H})}\|f\|_{\mathcal{H}}\|g\|_{\mathcal{H}}, \\
& \leq e^{k^{-1} 4 \pi^{2}}\left\|T S^{*} T^{-1}\right\|_{\mathcal{B}(\mathcal{H})}\|f\|_{\mathcal{H}}\left\|_{g}\right\|_{\mathcal{H}}, \quad f, g \in \operatorname{dom}\left(T^{2}\right), y \in \mathbb{R},
\end{aligned}
$$

using (2.9), and similarly,

$$
\begin{aligned}
& \left|\varphi_{k}(1+i y)\right|=e^{-k y^{2}}\left|\left(f, T^{1+2 i y} S T^{-1-2 i y} g\right)_{\mathcal{H}}\right| \\
& \quad=e^{-k y^{2}}\left|\left(T^{-2 i y} f,\left[T S T^{-1}\right] T^{-2 i y} g\right)_{\mathcal{H}}\right| \\
& \leq e^{k^{-1} 4 \pi^{2}}\left\|T S T^{-1}\right\|_{\mathcal{B}(\mathcal{H})}\|f\|_{\mathcal{H}}\|g\|_{\mathcal{H}}, \quad f, g \in \operatorname{dom}\left(T^{2}\right), y \in \mathbb{R} .
\end{aligned}
$$

In addition, one obtains

$$
\begin{aligned}
\left|\varphi_{k}(z)\right|= & e^{-k|\operatorname{Im}(z)|^{2}+k \operatorname{Re}(z)[\operatorname{Re}(z)-1]}\left|\left(T^{2} f, T^{2 z-3} S T^{-1-2 z} T^{2} g\right)_{\mathcal{H}}\right| \\
\leq & e^{-k|\operatorname{Im}(z)|^{2}+k \operatorname{Re}(z)[\operatorname{Re}(z)-1]+4 \pi|\operatorname{Im}(z)|} \\
& \times\left\|T^{2 \operatorname{Re}(z)-3}\right\|_{\mathcal{B}(\mathcal{H})}\left\|S T^{-1}\right\|_{\mathcal{B}(\mathcal{H})}\left\|T^{-2 \operatorname{Re}(z)}\right\|_{\mathcal{B}(\mathcal{H})}\left\|T^{2} f\right\|_{\mathcal{H}}\left\|T^{2} g\right\|_{\mathcal{H}} \\
\leq & e^{k^{-1} 4 \pi^{2}+k \operatorname{Re}(z)[\operatorname{Re}(z)-1]} \\
& \times\left\|T^{2 \operatorname{Re}(z)-3}\right\|_{\mathcal{B}(\mathcal{H})}\left\|S T^{-1}\right\|_{\mathcal{B}(\mathcal{H})}\left\|T^{-2 \operatorname{Re}(z)}\right\|_{\mathcal{B}(\mathcal{H})}\left\|T^{2} f\right\|_{\mathcal{H}}\left\|T^{2} g\right\|_{\mathcal{H}} \\
\leq & C, \quad f, g \in \operatorname{dom}\left(T^{2}\right), z \in \bar{\Sigma},
\end{aligned}
$$

for some finite constant $C=C(f, g, S, T)>0$, independent of $z \in \bar{\Sigma}$. 
Applying the Hadamard three-lines estimate (2.25) to $\varphi(\cdot)$ then yields

$$
\begin{array}{r}
\left|\varphi_{k}(z)\right| \leq e^{k^{-1} 4 \pi^{2}}\left\|T S^{*} T^{-1}\right\|_{\mathcal{B}(\mathcal{H})}^{1-\operatorname{Re}(z)}\left\|T S T^{-1}\right\|_{\mathcal{B}(\mathcal{H})}^{\operatorname{Re}(z)}\|f\|_{\mathcal{H}}\|g\|_{\mathcal{H}}, \\
f, g \in \operatorname{dom}\left(T^{2}\right), z \in \bar{\Sigma} .
\end{array}
$$

Taking $z=1 / 2$ in (2.37) implies

$$
\begin{array}{r}
\left|(f, S g)_{\mathcal{H}}\right| \leq e^{4^{-1} k+k^{-1} 4 \pi^{2}}\left\|T S^{*} T^{-1}\right\|_{\mathcal{B}(\mathcal{H})}^{1 / 2}\left\|T S T^{-1}\right\|_{\mathcal{B}(\mathcal{H})}^{1 / 2}\|f\|_{\mathcal{H}}\|g\|_{\mathcal{H}}, \\
f, g \in \operatorname{dom}\left(T^{2}\right) .
\end{array}
$$

Optimizing with respect to $k>0$ yields

$$
\left|(f, S g)_{\mathcal{H}}\right| \leq e^{2 \pi}\left\|T S^{*} T^{-1}\right\|_{\mathcal{B}(\mathcal{H})}^{1 / 2}\left\|T S T^{-1}\right\|_{\mathcal{B}(\mathcal{H})}^{1 / 2}\|f\|_{\mathcal{H}}\|g\|_{\mathcal{H}}, \quad f, g \in \operatorname{dom}\left(T^{2}\right) .
$$

Since $\operatorname{dom}\left(T^{2}\right)$ is dense in $\mathcal{H}$, this yields that $S$ is a bounded operator in $\mathcal{H}$. Employing that $S$ is closed in $\mathcal{H}$ finally proves $S \in \mathcal{B}(\mathcal{H})$ and hence the first estimate in (2.32).

If in addition, $T \geq 0$, we choose $k=0$ in (2.33) and then rely on equality (2.31) (as opposed to (2.30), which slightly simplifies the estimates (2.34)-(2.39), implying the second inequality in (2.32).

Remark 2.6. In the special case where $T$ is self-adjoint and $T \geq \varepsilon I_{\mathcal{H}}$ for some $\varepsilon>0$, there exists an alternative way of deriving the bound (2.32) by means of Proposition A.1 (2) proved by Lesch 22 in the context of closed, symmetric operators $S$. Indeed, an application of [22, Proposition A.1 (2)] with $S$ replaced by the symmetric, in fact, self-adjoint, $S^{*} S$ yields

$$
\begin{aligned}
\left\|S^{*} S\right\|_{\mathcal{B}(\mathcal{H})} & \leq\left\|T S^{*} S T^{-1}\right\|_{\mathcal{B}(\mathcal{H})}=\left\|\left(T S^{*} T^{-1}\right)\left(T S T^{-1}\right)\right\|_{\mathcal{B}(\mathcal{H})} \\
& \leq\left\|T S^{*} T^{-1}\right\|_{\mathcal{B}(\mathcal{H})}\left\|T S T^{-1}\right\|_{\mathcal{B}(\mathcal{H})} .
\end{aligned}
$$

Thus, $S^{*} S$ is bounded, so both $S$ and $S^{*}$ are bounded, and hence,

$$
\|S\|_{\mathcal{B}(\mathcal{H})}=\left\|S^{*}\right\|_{\mathcal{B}(\mathcal{H})}=\left\|\left(S^{*} S\right)\right\|_{\mathcal{B}(\mathcal{H})}^{1 / 2} \leq\left\|T S T^{-1}\right\|_{\mathcal{B}(\mathcal{H})}^{1 / 2}\left\|T S^{*} T^{-1}\right\|_{\mathcal{B}(\mathcal{H})}^{1 / 2} .
$$

Thus, in this special case one needs no additional arguments to prove Theorem 2.5 However, this type of argument does not apply to the remaining statements in this section.

Theorem 2.5 allows us to derive the following result.

Theorem 2.7. In addition to Hypothesis 2.1 suppose that $T \geq 0$. Then

$$
T^{-1 / 2} S T^{-1 / 2} \in \mathcal{B}(\mathcal{H}), \quad T^{-1 / 2} S^{*} T^{-1 / 2} \in \mathcal{B}(\mathcal{H}),
$$

and

$$
\left(T^{-1 / 2} S T^{-1 / 2}\right)^{*}=T^{-1 / 2} S^{*} T^{-1 / 2}, \quad\left(T^{-1 / 2} S^{*} T^{-1 / 2}\right)^{*}=T^{-1 / 2} S T^{-1 / 2} .
$$

Moreover,

$$
\left\|T^{-1 / 2} S T^{-1 / 2}\right\|_{\mathcal{B}(\mathcal{H})}=\left\|T^{-1 / 2} S^{*} T^{-1 / 2}\right\|_{\mathcal{B}(\mathcal{H})} \leq\left\|S T^{-1}\right\|_{\mathcal{B}(\mathcal{H})}^{1 / 2}\left\|S^{*} T^{-1}\right\|_{\mathcal{B}(\mathcal{H})}^{1 / 2} .
$$


Proof. The combined assumptions on $T$ actually yield $T \geq \varepsilon I_{\mathcal{H}}$ for some $\varepsilon>0$. (The condition $T \geq 0$ has inadvertently been omitted in [6, Theorem 4.1] and [22, Proposition A.1 (3)].) Introduce the operators $\widehat{S}$ and $\widehat{T}$ in $\mathcal{H}$ by

$$
\widehat{S}=T^{-1 / 2} S T^{-1 / 2}, \quad \widehat{T}=T^{1 / 2} .
$$

Then $\widehat{T}$ is self-adjoint and $\operatorname{dom}(\widehat{S}) \supseteq \operatorname{dom}\left(T^{1 / 2}\right)$, that is, $\operatorname{dom}(\widehat{S}) \supseteq \operatorname{dom}(\widehat{T})$ yields that $\widehat{S}$ is densely defined. Next, we note that by Remark 2.2 (ii),

$$
(\widehat{S})^{*}=\left(T^{-1 / 2}\left[S T^{-1 / 2}\right]\right)^{*}=\left[S T^{-1 / 2}\right]^{*} T^{-1 / 2} \supseteq T^{-1 / 2} S^{*} T^{-1 / 2},
$$

and hence $\operatorname{dom}\left((\widehat{S})^{*}\right) \supseteq \operatorname{dom}(\widehat{T})$. Moreover, since

$$
\widehat{T} \widehat{S}(\widehat{T})^{-1}=S T^{-1} \in \mathcal{B}(\mathcal{H}), \quad \widehat{T}(\widehat{S})^{*}(\widehat{T})^{-1} \supseteq S^{*} T^{-1} \in \mathcal{B}(\mathcal{H}),
$$

by Hypothesis 2.1 (resp., (2.2)), one also infers

$$
\widehat{T}(\widehat{S})^{*}(\widehat{T})^{-1}=S^{*} T^{-1} \in \mathcal{B}(\mathcal{H}) .
$$

Thus, Theorem 2.5 applies to $\widehat{S}$ and $\widehat{T}$ and hence $\widehat{S},(\widehat{S})^{*} \in \mathcal{B}(\mathcal{H})$, as well as

$$
\begin{aligned}
\|\widehat{S}\|_{\mathcal{B}(\mathcal{H})}=\left\|(\widehat{S})^{*}\right\|_{\mathcal{B}(\mathcal{H})} & \leq\left\|\widehat{T} \widehat{S}(\widehat{T})^{-1}\right\|_{\mathcal{B}(\mathcal{H})}^{1 / 2}\left\|\widehat{T}(\widehat{S})^{*}(\widehat{T})^{-1}\right\|_{\mathcal{B}(\mathcal{H})}^{1 / 2} \\
& =\left\|S T^{-1}\right\|_{\mathcal{B}(\mathcal{H})}^{1 / 2}\left\|S^{*} T^{-1}\right\|_{\mathcal{B}(\mathcal{H})}^{1 / 2} .
\end{aligned}
$$

Since our hypotheses are symmetric with respect to $S$ and $S^{*}$, interchanging $S$ and $S^{*}$, repeating (2.45)-(2.49) with $\widehat{S}$ replaced by

$$
\widetilde{S}=T^{-1 / 2} S^{*} T^{-1 / 2},
$$

then also yields $(\widetilde{S})^{*} \supseteq T^{-1 / 2} S T^{-1 / 2}=\widehat{S}$. Since $\widehat{S} \in \mathcal{B}(\mathcal{H})$, one concludes that $(\widetilde{S})^{*}=\widehat{S}$. Applying Theorem 2.5 to $\widetilde{S}$ and $\widehat{T}$ then yields $\widetilde{S} \in \mathcal{B}(\mathcal{H})$ and hence also $(\widehat{S})^{*}=\widetilde{S}$, completing the proof.

In the special case where $S$ is symmetric in $\mathcal{H}, S \subseteq S^{*}$, and $T \geq 0$ (actually, $T \geq \varepsilon I_{\mathcal{H}}$ for some $\varepsilon>0$ as also the condition $T^{-1} \in \mathcal{B}(\mathcal{H})$ is involved), nearly all the results of this section up to now (as well as the basic strategy of proofs employed), appeared in Lesch [22, Appendix A]. We emphasize, however, that some of these results, especially, Theorem 2.7. were previously derived in 1988 by Huang [14, Lemma 2.1.(b)]. In fact, combining the spectral theorem for $T$ and the three-lines theorem, Huang arrives at an extension of Theorem 2.7 involving fractional powers of $T^{\alpha} \alpha \in[1 / 2,1]$ on the right-hand side of (2.44).

Next, we recall the generalized polar decomposition for densely defined closed operators $S$ in $\mathcal{H}$ derived in [7,

$$
S=\left|S^{*}\right|^{\alpha} U_{S}|S|^{1-\alpha}, \quad \alpha \in[0,1],
$$

where $U_{S}$ denotes the partial isometry in $\mathcal{H}$ associated with the standard polar decomposition $S=U_{S}|S|$, and $|S|=\left(S^{*} S\right)^{1 / 2}$ (and we interpret $|S|^{0}=I_{\mathcal{H}}$ in this particular context).

We will employ (2.51) (and its analog for $S^{*}$ ) to prove the following result:

Theorem 2.8. Assume Hypothesis 2.1. Then $T^{-z} S T^{-1+z}, z \in \bar{\Sigma}$, defined on $\operatorname{dom}(T)$, is closable in $\mathcal{H}$, and

$$
\overline{T^{-z} S T^{-1+z}}=T^{-i \operatorname{Im}(z)}\left[\left|S^{*}\right|^{\operatorname{Re}(z)} T^{-\operatorname{Re}(z)}\right]^{*} U_{S}
$$




$$
\times|S|^{1-\operatorname{Re}(z)} T^{-1+\operatorname{Re}(z)} T^{i \operatorname{Im}(z)} \in \mathcal{B}(\mathcal{H}), \quad z \in \bar{\Sigma} .
$$

In addition, given $k \in(0, \infty)$, one obtains

$$
\begin{aligned}
\left\|\overline{T^{-z} S T^{-1+z}}\right\|_{\mathcal{B}(\mathcal{H})} \leq & \left\|S T^{-1}\right\|_{\mathcal{B}(\mathcal{H})}^{1-\operatorname{Re}(z)}\left\|S^{*} T^{-1}\right\|_{\mathcal{B}(\mathcal{H})}^{\operatorname{Re}(z)} \\
& \times\left\{\begin{array}{l}
e^{k|\operatorname{Im}(z)|^{2}+k \operatorname{Re}(z)[1-\operatorname{Re}(z)]+k^{-1} \pi^{2}}, \quad z \in \bar{\Sigma}, \\
1, \text { if } T \geq 0,
\end{array}\right.
\end{aligned}
$$

and

$$
\left\|\overline{T^{-x} S T^{-1+x}}\right\|_{\mathcal{B}(\mathcal{H})} \leq\left\|S T^{-1}\right\|_{\mathcal{B}(\mathcal{H})}^{1-x}\left\|S^{*} T^{-1}\right\|_{\mathcal{B}(\mathcal{H})}^{x}\left\{\begin{array}{l}
e^{2 \pi[x(1-x)]^{1 / 2}} \\
1, \text { if } T \geq 0,
\end{array} \quad x \in[0,1] .\right.
$$

In particular, assuming $T \geq 0$ and taking $x=1 / 2$ in (2.54) one recovers the estimate (2.44) (in this particular case the operator closure sign in (2.53) is superfluous since $T^{-1 / 2} S T^{-1 / 2} \in \mathcal{B}(\mathcal{H})$ by $(2.42)$ ).

Proof. We start by noting that $\operatorname{dom}(S) \supseteq \operatorname{dom}(T)$ (together with $S$ and $T$ closed by hypothesis) implies that $S$ is relatively bounded with respect to $T$ and hence there exist $a>0$ and $b>0$ such that

$$
\begin{aligned}
\||S| f\|_{\mathcal{H}}^{2}=\|S f\|_{\mathcal{H}}^{2} & \leq a^{2}\|T f\|_{\mathcal{H}}^{2}+b^{2}\|f\|_{\mathcal{H}}^{2}=a^{2}\||T| f\|_{\mathcal{H}}^{2}+b^{2}\|f\|_{\mathcal{H}}^{2} \\
& =\left\|\left[a^{2}|T|^{2}+b^{2}\right]^{1 / 2} f\right\|_{\mathcal{H}}^{2}, \quad f \in \operatorname{dom}(T)=\operatorname{dom}(|T|) .
\end{aligned}
$$

Thus, applying Heinz's inequality (cf. [5, Sect. 3.2.1], [12, Theorem 3], [15], 21, Theorem IV.1.11], [26]), one infers that

$$
\operatorname{dom}\left(|S|^{\alpha}\right) \supseteq \operatorname{dom}\left(\left(a^{2}|T|^{2}+b^{2}\right)^{\alpha / 2}\right)=\operatorname{dom}\left(|T|^{\alpha}\right), \quad \alpha \in[0,1]
$$

and

$$
\left\||S|^{\alpha}\left[a^{2}|T|^{2}+b^{2}\right]^{-\alpha / 2} h\right\|_{\mathcal{H}} \leq\|h\|_{\mathcal{H}}^{2}, \quad h \in \mathcal{H}, \alpha \in[0,1] .
$$

Similarly, interchanging $S$ and $S^{*}$, one obtains

$$
\operatorname{dom}\left(\left|S^{*}\right|^{\alpha}\right) \supseteq \operatorname{dom}\left(|T|^{\alpha}\right), \quad \alpha \in[0,1],
$$

and

$$
\left\|\left|S^{*}\right|^{\alpha}\left[\widetilde{a}^{2}|T|^{2}+\widetilde{b}^{2}\right]^{-\alpha / 2} h\right\|_{\mathcal{H}} \leq\|h\|_{\mathcal{H}}^{2}, \quad h \in \mathcal{H}, \alpha \in[0,1],
$$

for appropriate $\widetilde{a}>0, \widetilde{b}>0$. Applying (2.51) to $S$ (with $\alpha=\operatorname{Re}(z)$ ), and using (2.56) and (2.58), one concludes that

$$
\begin{aligned}
& T^{-z} S T^{-1+z}=T^{-z}\left|S^{*}\right|^{\operatorname{Re}(z)} U_{S}|S|^{1-\operatorname{Re}(z)} T^{-1+z} \\
& \quad \subseteq\left[\left|S^{*}\right|^{\operatorname{Re}(z)} T^{-\bar{z}}\right]^{*} U_{S}\left[|S|^{1-\operatorname{Re}(z)} T^{-1+z}\right] \\
& \quad=T^{-i \operatorname{Im}(z)}\left(\left|S^{*}\right|^{\operatorname{Re}(z)} T^{-\operatorname{Re}(z)}\right)^{*} U_{S}|S|^{1-\operatorname{Re}(z)} T^{-1+\operatorname{Re}(z)} T^{i \operatorname{Im}(z)} \in \mathcal{B}(\mathcal{H}), \quad z \in \bar{\Sigma},
\end{aligned}
$$

proving (2.52).

Next, one defines (repeatedly employing below the fact that for a closable operator $A, \bar{A}$ is an extension of $A$ )

$$
\begin{array}{r}
\phi_{k}(z)=e^{k z(z-1)}\left(T f, T^{-1-z} S T^{-2+z} T g\right)_{\mathcal{H}}=e^{k z(z-1)}\left(f, \overline{T^{-z} S T^{-1+z}} g\right)_{\mathcal{H}}, \\
f, g \in \operatorname{dom}(T), z \in \Sigma, k \in[0, \infty) .
\end{array}
$$

Again we primarily focus on the case where $T$ is merely self-adjoint and hence choose $k>0$ and employ the estimate (2.30) in the following. 
One estimates

$$
\begin{aligned}
|\phi(i y)|= & e^{-k y^{2}}\left|\left(T^{i y} f, S T^{-1} T^{i y} g\right)_{\mathcal{H}}\right| \leq e^{-k y^{2}+2 \pi|y|}\left\|S T^{-1}\right\|_{\mathcal{B}(\mathcal{H})}\|f\|_{\mathcal{H}}\|g\|_{\mathcal{H}} \\
\leq & e^{k^{-1} \pi^{2}}\left\|S T^{-1}\right\|_{\mathcal{B}(\mathcal{H})}\|f\|_{\mathcal{H}}\|g\|_{\mathcal{H}}, \quad y \in \mathbb{R}, \\
|\phi(1+i y)|= & e^{-k y^{2}}\left|\left(T^{i y} f, \overline{T^{-1} S T^{i y}} g\right)_{\mathcal{H}}\right| \leq e^{-k y^{2}+2 \pi|y|}\left\|\overline{T^{-1} S}\right\|_{\mathcal{B}(\mathcal{H})}\|f\|_{\mathcal{H}}\|g\|_{\mathcal{H}} \\
\leq & e^{k^{-1} \pi^{2}}\left\|S^{*} T^{-1}\right\|_{\mathcal{B}(\mathcal{H})}\|f\|_{\mathcal{H}}\|g\|_{\mathcal{H}}, \quad y \in \mathbb{R}, \\
|\phi(z)|= & e^{-k|\operatorname{Im}(z)|^{2}+k \operatorname{Re}(z)[\operatorname{Re}(z)-1]}\left|\left(T f, T^{-1-z} S T^{-2+z} T g\right)_{\mathcal{H}}\right| \\
\leq & e^{-k|\operatorname{Im}(z)|^{2}+k \operatorname{Re}(z)[\operatorname{Re}(z)-1]+2 \pi|\operatorname{Im}(z)|}\left\|T^{-1-\operatorname{Re}(z)}\right\|_{\mathcal{B}(\mathcal{H})} \\
& \times\left\|S T^{-1}\right\|_{\mathcal{B}(\mathcal{H})}\left\|T^{-1+\operatorname{Re}(z)}\right\|_{\mathcal{B}(\mathcal{H})}\|T f\|_{\mathcal{H}}\|T g\|_{\mathcal{H}} \\
\leq & C, \quad f, g \in \operatorname{dom}(T), z \in \Sigma
\end{aligned}
$$

where $C=C(f, g, S, T)>0$ is a finite constant, independent of $z \in \bar{\Sigma}$.

Applying the Hadamard three-lines estimate (2.25) to $\phi(\cdot)$ then yields the first estimate in (2.53) since $\operatorname{dom}(T)$ is dense in $\mathcal{H}$ and $\overline{T^{-z} S T^{-1+z}} \in \mathcal{B}(\mathcal{H}), z \in \bar{\Sigma}$, by (2.52).

If in addition $T \geq 0$, one chooses $k=0$ in (2.61) and uses (2.31) (instead of (2.30) ) to arrive at the second estimate in (2.53).

We emphasize that the case $T \geq 0$ (actually, $T \geq \varepsilon I_{\mathcal{H}}$ for some $\varepsilon>0$ ) in the estimate (2.54) was also derived by Huang [14, Lemma 2.1.(a)].

The results provided thus far naturally extend to the situation where $T^{-z} S T^{-1+z}$ is replaced by $T_{2}^{-z} S T_{1}^{-1+z}$ for two self-adjoint operators $T_{j}$ in $\mathcal{H}_{j}, j=1,2$. As an example, we now illustrate this in the context of Theorem 2.8 .

Hypothesis 2.9. Assume that $T_{j}$ are self-adjoint operators in $\mathcal{H}_{j}$ with $T_{j}^{-1} \in$ $\mathcal{B}\left(\mathcal{H}_{j}\right), j=1,2$. In addition, suppose that $S$ is a closed operator mapping $\operatorname{dom}(S) \subseteq$ $\mathcal{H}_{1}$ into $\mathcal{H}_{2}$ satisfying

$$
\operatorname{dom}(S) \supseteq \operatorname{dom}\left(T_{1}\right) \text { and } \operatorname{dom}\left(S^{*}\right) \supseteq \operatorname{dom}\left(T_{2}\right) .
$$

In particular, Hypothesis 2.9 implies that

$$
S T_{1}^{-1} \in \mathcal{B}\left(\mathcal{H}_{1}, \mathcal{H}_{2}\right), \quad S^{*} T_{2}^{-1} \in \mathcal{B}\left(\mathcal{H}_{2}, \mathcal{H}_{1}\right) .
$$

Assuming Hypothesis 2.9 one obtains the following corollary of Theorem 2.8

Corollary 2.10. Assume Hypothesis 2.9 , Then $T_{2}^{-z} S T_{1}^{-1+z}$ defined on $\operatorname{dom}\left(T_{1}\right)$, $z \in \bar{\Sigma}$, is closable, and

$$
\begin{aligned}
\overline{T_{2}^{-z} S T_{1}^{-1+z}}= & T_{2}^{-i \operatorname{Im}(z)}\left[\left|S^{*}\right|^{\operatorname{Re}(z)} T_{2}^{-\operatorname{Re}(z)}\right]^{*} U_{S} \\
& \times|S|^{1-\operatorname{Re}(z)} T_{1}^{-1+\operatorname{Re}(z)} T_{1}^{i \operatorname{Im}(z)} \in \mathcal{B}\left(\mathcal{H}_{1}, \mathcal{H}_{2}\right), \quad z \in \bar{\Sigma} .
\end{aligned}
$$

In addition, given $k \in(0, \infty)$, one obtains

$$
\begin{aligned}
& \left\|\overline{T_{2}^{-z} S T_{1}^{-1+z}}\right\|_{\mathcal{B}\left(\mathcal{H}_{1}, \mathcal{H}_{2}\right)} \leq\left\|S T_{1}^{-1}\right\|_{\mathcal{B}\left(\mathcal{H}_{1}, \mathcal{H}_{2}\right)}^{1-\operatorname{Re}\left(S^{*} T_{2}^{-1} \|_{\mathcal{B}\left(\mathcal{H}_{2}, \mathcal{H}_{1}\right)}^{\operatorname{Re}(z)}\right.} \\
& \quad \times\left\{\begin{array}{l}
e^{k|\operatorname{Im}(z)|^{2}+k \operatorname{Re}(z)[1-\operatorname{Re}(z)]+k^{-1} \pi^{2}}, \\
e^{k|\operatorname{Im}(z)|^{2}+k \operatorname{Re}(z)[1-\operatorname{Re}(z)]+(4 k)^{-1} \pi^{2}}, \text { if } T_{1} \geq 0, \text { or } T_{2} \geq 0, \quad z \in \bar{\Sigma}, \\
1, \text { if } T_{j} \geq 0, j=1,2,
\end{array}\right.
\end{aligned}
$$


and

$$
\begin{aligned}
& \left\|\overline{T_{2}^{-x} S T_{1}^{-1+x}}\right\|_{\mathcal{B}\left(\mathcal{H}_{1}, \mathcal{H}_{2}\right)} \leq\left\|S T_{1}^{-1}\right\|_{\mathcal{B}\left(\mathcal{H}_{1}, \mathcal{H}_{2}\right)}^{1-x}\left\|S^{*} T_{2}^{-1}\right\|_{\mathcal{B}\left(\mathcal{H}_{2}, \mathcal{H}_{1}\right)}^{x} \\
& \quad \times\left\{\begin{array}{l}
e^{2 \pi[x(1-x)]^{1 / 2}}, \\
e^{\pi[x(1-x)]^{1 / 2}}, \text { if } T_{1} \geq 0, \text { or } T_{2} \geq 0, \\
1, \text { if } T_{j} \geq 0, j=1,2,
\end{array}\right.
\end{aligned}
$$

Proof. Consider $\mathcal{H}:=\mathcal{H}_{1} \oplus \mathcal{H}_{2}$ and introduce

$$
\begin{aligned}
\mathbf{S} & =\left(\begin{array}{cc}
0 & 0 \\
S & 0
\end{array}\right), \quad \operatorname{dom}(\mathbf{S})=\operatorname{dom}(S) \oplus \mathcal{H}_{2}, \\
\mathbf{S}^{*} & =\left(\begin{array}{cc}
0 & S^{*} \\
0 & 0
\end{array}\right), \quad \operatorname{dom}\left(\mathbf{S}^{*}\right)=\mathcal{H}_{1} \oplus \operatorname{dom}\left(S^{*}\right),
\end{aligned}
$$

and

$$
\mathbf{T}=\left(\begin{array}{cc}
T_{1} & 0 \\
0 & T_{2}
\end{array}\right), \quad \operatorname{dom}(\mathbf{T})=\operatorname{dom}\left(T_{1}\right) \oplus \operatorname{dom}\left(T_{2}\right)
$$

Then $\mathbf{S}$ is a closed operator in $\mathcal{H}$ and $\mathbf{T}$ is a self-adjoint operator in $\mathcal{H}$ with bounded inverse given by

$$
\mathbf{T}^{-1}=\left(\begin{array}{cc}
T_{1}^{-1} & 0 \\
0 & T_{2}^{-1}
\end{array}\right) \in \mathcal{B}(\mathcal{H})
$$

Moreover,

$$
\operatorname{dom}(\mathbf{S}) \cap \operatorname{dom}\left(\mathbf{S}^{*}\right)=\operatorname{dom}(S) \oplus \operatorname{dom}\left(S^{*}\right) \supseteq \operatorname{dom}\left(T_{1}\right) \oplus \operatorname{dom}\left(T_{2}\right)=\operatorname{dom}(\mathbf{T}),
$$

that is, the pair $(\mathbf{S}, \mathbf{T})$ satisfies Hypothesis 2.1 .

Thus,

$$
\mathbf{S T}^{-1}=\left(\begin{array}{cc}
0 & 0 \\
S T_{1}^{-1} & 0
\end{array}\right) \in \mathcal{B}(\mathcal{H}), \quad \mathbf{S}^{*} \mathbf{T}^{-1}=\left(\begin{array}{cc}
0 & S^{*} T_{2}^{-1} \\
0 & 0
\end{array}\right) \in \mathcal{B}(\mathcal{H})
$$

and

$$
\left\|\mathbf{S} \mathbf{T}^{-1}\right\|_{\mathcal{B}(\mathcal{H})}=\left\|S T_{1}^{-1}\right\|_{\mathcal{B}\left(\mathcal{H}_{1}, \mathcal{H}_{2}\right)}, \quad\left\|\mathbf{S}^{*} \mathbf{T}^{-1}\right\|_{\mathcal{B}(\mathcal{H})}=\left\|S^{*} T_{2}^{-1}\right\|_{\mathcal{B}\left(\mathcal{H}_{2}, \mathcal{H}_{1}\right)} .
$$

Applying Theorem 2.8 to the pair (S, T), one obtains (2.68) and (2.69).

In the special case, where $\mathcal{H}_{1}=\mathcal{H}_{2}=\mathcal{H}, S$ and $T_{1}, T_{2} \geqslant 0$ are bounded linear operators in $\mathcal{H}$, the third estimate (2.69) recovers Lemma 25 in 4 . On the other hand, the case $x \in[0,1]$ in the first estimate in (2.69) is a special case of (4.32), which in turn is recorded in [42, Lemma 16.3].

For connections with the generalized Heinz inequality and bounds on operators of the type $T_{2}^{-x} S T_{1}^{-x}, x \in[0,1]$, under various conditions on $S$ and $T_{j}, j=1,2$, we also refer to [14, Lemma 2.1], [16, Theorem 3], [18, Theorem 6], and the references cited therein. In particular, [14, Lemma 2.1] predates and extends 22, Proposition A.1.(3)]. For additional variants on the Heinz inequality we refer, for instance, to [27, Lemma 11, Remark 12], and especially, to [5, Sect. 3.12] and the detailed bibliography collected therein. For interesting extensions of the Heinz inequality employing operator monotone functions we also refer to [37, 39], 40]. (An exhaustive list of references on (extensions of) the Heinz inequality is beyond the scope of this short paper due to the enormous amount of literature on this subject.) 


\section{Interpolation And Trace Ideals Revisited}

In this section we recall a powerful result on interpolation theory in connection with linear operators in the trace ideal spaces $\mathcal{B}_{p}(\mathcal{H}), p \in[1, \infty)$, originally due to I. C. Gohberg, M. G. Krein, and S. G. Krein (cf. [8, p. 139]) and then apply it to the types of operators studied in Section 2, For background on trace ideals we refer to [8, Ch. I-III], 32, 33, Chs. 1-3]

In particular, we will dwell a bit on a particular case omitted in the discussion of Gohberg and Krein [8, Theorem III.13.1] (see also [9, Theorem III.5.1]):

Theorem 3.1 ([8, Theorem III.13.1 (see also 9], Theorem III.5.1)).

Let $p_{0}, p_{1} \in[1, \infty), p_{0} \leq p_{1}$, and suppose that $A(z) \in \mathcal{B}(\mathcal{H}), z \in \bar{\Sigma}$, and that $A(\cdot)$ is analytic on $\Sigma$. Assume that for some $C_{0}, C_{1} \in(0, \infty)$,

$$
\|A(i y)\|_{\mathcal{B}_{p_{0}}(\mathcal{H})} \leq C_{0}, \quad\|A(1+i y)\|_{\mathcal{B}_{p_{1}}(\mathcal{H})} \leq C_{1}, \quad y \in \mathbb{R}
$$

and suppose that for all $f, g \in \mathcal{H}$, there exist $C_{f, g} \in \mathbb{R}$ and $a_{f, g} \in[0, \pi)$, such that

$$
\sup _{z \in \Sigma}\left[e^{-a_{f, g}|\operatorname{Im}(z)|} \ln \left(\left|(f, A(z) g)_{\mathcal{H}}\right|\right)\right] \leq C_{f, g} .
$$

Then

$$
A(z) \in \mathcal{B}_{p_{z}}(\mathcal{H}), \quad \frac{1}{p_{z}}=\frac{1-\operatorname{Re}(z)}{p_{0}}+\frac{\operatorname{Re}(z)}{p_{1}}, \quad z \in \bar{\Sigma},
$$

and

$$
\|A(z)\|_{\mathcal{B}_{p_{z}}(\mathcal{H})} \leq C_{0}^{1-\operatorname{Re}(z)} C_{1}^{\operatorname{Re}(z)}, \quad z \in \bar{\Sigma} .
$$

The estimate (3.4) remains valid for $p_{0} \in[1, \infty)$ and $p_{1}=\infty$ in (3.1) and (3.3). In particular, if $p_{0}=p_{1} \in[1, \infty)$, then

$$
\|A(z)\|_{\mathcal{B}_{p_{0}}(\mathcal{H})} \leq C_{0}^{1-\operatorname{Re}(z)} C_{1}^{\operatorname{Re}(z)}, \quad z \in \bar{\Sigma} .
$$

Proof. Theorem 3.1 and its proof is presented by Gohberg and Krein in 8, Theorem III.13.1] under the additional assumption that $p_{0}<p_{1}$. (Moreover, this additional restriction is repeated in [9, Theorem III.5.1], where the result is stated without proof.) However, their proof extends to special case where $p_{0}=p_{1} \in[1, \infty)$ without any difficulties, in fact, it even simplifies a bit. For the convenience of the reader we now present the proof in this particular situation:

Let $F \in \mathcal{B}(\mathcal{H})$ be a finite-rank operator and suppose that

$$
\begin{aligned}
& \|F\|_{\mathcal{B}_{p_{0}^{\prime}}(\mathcal{H})}=1, \quad p_{0}^{-1}+\left(p_{0}^{\prime}\right)^{-1}=1, \text { if } p_{0} \in(1, \infty), \\
& \|F\|_{\mathcal{B}(\mathcal{H})}=1 \text {, if } p_{0}=1,
\end{aligned}
$$

and consider the function

$$
\varphi(z)=\operatorname{tr}(A(z) F), \quad z \in \bar{\Sigma}
$$

By the assumptions on $A(\cdot), \varphi(\cdot)$ is analytic on $\Sigma$ and

$$
\sup _{z \in \Sigma}\left[e^{-a|\operatorname{Im}(z)|} \ln (|\varphi(z)|)\right] \leq C
$$

for some $a=a(F) \in[0, \pi)$ and $C=C(F) \in \mathbb{R}$. In addition, one estimates

$$
\begin{aligned}
|\varphi(i y)| & \leq\|A(i y) F\|_{\mathcal{B}_{1}(\mathcal{H})} \leq\|A(i y)\|_{\mathcal{B}_{p_{0}}(\mathcal{H})}\|F\|_{\mathcal{B}_{p_{0}^{\prime}}(\mathcal{H})} \leq C_{0}, \quad y \in \mathbb{R}, \quad(3.9 \\
|\varphi(1+i y)| & \leq\|A(1+i y) F\|_{\mathcal{B}_{1}(\mathcal{H})} \leq\|A(1+i y)\|_{\mathcal{B}_{p_{0}}(\mathcal{H})}\|F\|_{\mathcal{B}_{p_{0}^{\prime}}(\mathcal{H})} \leq C_{1}, \quad y \in \mathbb{R}
\end{aligned}
$$


Applying Hadamard's three-lines estimate (2.25) to $\varphi(\cdot)$ then yields

$$
|\varphi(z)|=|\operatorname{tr}(A(z) F)| \leq C_{0}^{1-\operatorname{Re}(z)} C_{1}^{\operatorname{Re}(z)}, \quad z \in \bar{\Sigma} .
$$

Next, denoting by $\mathcal{F}(\mathcal{H})$ the set of all finite-rank operators in $\mathcal{H}$, we recall that $B \in \mathcal{B}_{p_{0}}(\mathcal{H})$ if and only if the number $\|B\|_{\mathcal{B}_{p_{0}}(\mathcal{H})}$ is finite, where

$$
\|B\|_{\mathcal{B}_{p_{0}}(\mathcal{H})}= \begin{cases}\sup _{0 \neq F \in \mathcal{F}(\mathcal{H})}|\operatorname{tr}(B F)| /\|F\|_{\mathcal{B}_{p_{0}^{\prime}}(\mathcal{H})}, & p_{0} \in(1, \infty), \\ \sup _{0 \neq F \in \mathcal{F}(\mathcal{H})}|\operatorname{tr}(B F)| /\|F\|_{\mathcal{B}(\mathcal{H})}, & p_{0}=1\end{cases}
$$

(cf. [8, Lemma III.12.1]). Thus, (3.11) implies (3.5) due to the normalization in (3.6).

Alternatively, Theorem 3.1 follows from combining Theorems IX.20, IX.22, and Proposition 8 in [28].

Next we prove a trace ideal analog of Theorem 2.8, using Theorem 3.1

Theorem 3.2. In addition to Hypothesis 2.1, let $p \in[1, \infty)$, and assume that

$$
S T^{-1} \in \mathcal{B}_{p}(\mathcal{H}), \quad S^{*} T^{-1} \in \mathcal{B}_{p}(\mathcal{H}) .
$$

Then $T^{-z} S T^{-1+z}, z \in \bar{\Sigma}$, defined on $\operatorname{dom}(T)$, is closable in $\mathcal{H}$, and

$$
\overline{T^{-z} S T^{-1+z}} \in \mathcal{B}_{p}(\mathcal{H}), \quad z \in \bar{\Sigma} \text {. }
$$

In addition, given $k \in(0, \infty)$, one obtains

$$
\begin{aligned}
\left\|\overline{T^{-z} S T^{-1+z}}\right\|_{\mathcal{B}_{p}(\mathcal{H})} \leq & \left\|S T^{-1}\right\|_{\mathcal{B}_{p}(\mathcal{H})}^{1-\operatorname{Re}(z)}\left\|S^{*} T^{-1}\right\|_{\mathcal{B}_{p}(\mathcal{H})}^{\operatorname{Re}(z)} \\
& \times\left\{\begin{array}{l}
e^{k|\operatorname{Im}(z)|^{2}+k \operatorname{Re}(z)[1-\operatorname{Re}(z)]+k^{-1} \pi^{2}}, \quad z \in \bar{\Sigma}, \\
1, \text { if } T \geq 0,
\end{array}\right.
\end{aligned}
$$

and

$$
\left\|\overline{T^{-x} S T^{-1+x}}\right\|_{\mathcal{B}_{p}(\mathcal{H})} \leq\left\|S T^{-1}\right\|_{\mathcal{B}_{p}(\mathcal{H})}^{1-x}\left\|S^{*} T^{-1}\right\|_{\mathcal{B}_{p}(\mathcal{H})}^{x}\left\{\begin{array}{l}
e^{2 \pi[x(1-x)]^{1 / 2}}, \\
1, \text { if } T \geq 0,
\end{array} \quad x \in[0,1] .\right.
$$

In particular, assuming $T \geq 0$ and taking $x=1 / 2$ in (3.16) one obtains

$$
T^{-1 / 2} S T^{-1 / 2}=\left(T^{-1 / 2} S^{*} T^{-1 / 2}\right)^{*} \in \mathcal{B}_{p}(\mathcal{H}),
$$

and

$$
\left\|T^{-1 / 2} S T^{-1 / 2}\right\|_{\mathcal{B}_{p}(\mathcal{H})}=\left\|T^{-1 / 2} S^{*} T^{-1 / 2}\right\|_{\mathcal{B}_{p}(\mathcal{H})} \leq\left\|S T^{-1}\right\|_{\mathcal{B}_{p}(\mathcal{H})}^{1 / 2}\left\|S^{*} T^{-1}\right\|_{\mathcal{B}_{p}(\mathcal{H})}^{1 / 2} .
$$

Proof. First we note that Theorem 2.8 applies and hence (2.52), (2.53) are at our disposal. Next, we introduce

$$
A(z)=e^{k z(z-1)} \overline{T^{-z} S T^{-1+z}}, \quad z \in \bar{\Sigma}, k \in[0, \infty),
$$

and focus again on $k>0$ first.

Employing (2.30) one estimates

$$
\begin{aligned}
& \|A(i y)\|_{\mathcal{B}_{p}(\mathcal{H})}=e^{-k y^{2}}\left\|T^{-i y} S T^{-1} T^{i y}\right\|_{\mathcal{B}_{p}(\mathcal{H})} \leq e^{-k y^{2}+2 \pi|y|}\left\|S T^{-1}\right\|_{\mathcal{B}_{p}(\mathcal{H})} \\
& \quad \leq e^{k^{-1} \pi^{2}}\left\|S T^{-1}\right\|_{\mathcal{B}_{p}(\mathcal{H})}, \quad y \in \mathbb{R}, \\
& \|A(1+i y)\|_{\mathcal{B}_{p}(\mathcal{H})}=e^{-k y^{2}}\left\|\overline{T^{-1-i y} S T^{i y}}\right\|_{\mathcal{B}_{p}(\mathcal{H})}=e^{-k y^{2}}\left\|T^{-i y}\left(S^{*} T^{-1}\right)^{*} T^{i y}\right\|_{\mathcal{B}_{p}(\mathcal{H})}
\end{aligned}
$$




$$
\begin{aligned}
= & e^{k^{-1} \pi^{2}}\left\|S^{*} T^{-1}\right\|_{\mathcal{B}_{p}(\mathcal{H})}, \quad y \in \mathbb{R}, \\
\| A(z) & \|_{\mathcal{B}(\mathcal{H})}=e^{-k|\operatorname{Im}(z)|^{2}+k \operatorname{Re}(z)[\operatorname{Re}(z)-1]} \\
& \times\left\|T^{-i \operatorname{Im}(z)}\left(\left|S^{*}\right|^{\operatorname{Re}(z)} T^{-\operatorname{Re}(z)}\right)^{*} U_{S}|S|^{1-\operatorname{Re}(z)} T^{-1+\operatorname{Re}(z)} T^{i \operatorname{Im}(z)}\right\|_{\mathcal{B}(\mathcal{H})} \\
\leq & e^{-k|\operatorname{Im}(z)|^{2}+k \operatorname{Re}(z)[\operatorname{Re}(z)-1]+2 \pi|\operatorname{Im}(z)|} \\
& \times\left\|\left|S^{*}\right|^{\operatorname{Re}(z)} T^{-\operatorname{Re}(z)}\right\|_{\mathcal{B}(\mathcal{H})}\left\||S|^{1-\operatorname{Re}(z)} T^{-1+\operatorname{Re}(z)}\right\|_{\mathcal{B}(\mathcal{H})} \\
\leq & C, \quad z \in \bar{\Sigma},
\end{aligned}
$$

where $C=C(S, T)>0$ is a finite constant, independent of $z \in \bar{\Sigma}$, employing (2.57) and (2.59). Here again we used the generalized polar decomposition (2.51) for $S$ (with $\alpha=\operatorname{Re}(z)$ ).

Applying the Hadamard three-lines estimate (3.5) to $A(\cdot)$ then yields the first relation in (3.14) and the estimate (3.15).

In the special case where $T \geq 0$ and $S=S^{*} \in \mathcal{B}(\mathcal{H})$, the second estimate (3.16) recovers the result [35, Lemma 15] (see also [23, Lemma 5.10]). For applications of (3.16) to scattering theory we refer, for instance, to [29, Appendix 1].

Corollary 3.3. In addition to Hypothesis 2.9, let $p \in[1, \infty)$ and assume that

$$
S T_{1}^{-1} \in \mathcal{B}_{p}\left(\mathcal{H}_{1}, \mathcal{H}_{2}\right), \quad S^{*} T_{2}^{-1} \in \mathcal{B}_{p}\left(\mathcal{H}_{2}, \mathcal{H}_{1}\right) .
$$

Then $T_{2}^{-z} S T_{1}^{-1+z}$ defined on $\operatorname{dom}\left(T_{1}\right), z \in \bar{\Sigma}$, is closable, and

$$
\overline{T_{2}^{-z} S T_{1}^{-1+z}} \in \mathcal{B}_{p}\left(\mathcal{H}_{1}, \mathcal{H}_{2}\right), \quad z \in \bar{\Sigma} .
$$

In addition, given $k \in(0, \infty)$, one obtains

$$
\begin{aligned}
& \left\|\overline{T_{2}^{-z} S T_{1}^{-1+z}}\right\|_{\mathcal{B}_{p}\left(\mathcal{H}_{1}, \mathcal{H}_{2}\right)} \leq\left\|S T_{1}^{-1}\right\|_{\mathcal{B}_{p}\left(\mathcal{H}_{1}, \mathcal{H}_{2}\right)}^{1-\operatorname{Re}(z)}\left\|S^{*} T_{2}^{-1}\right\|_{\mathcal{B}_{p}\left(\mathcal{H}_{2}, \mathcal{H}_{1}\right)}^{\operatorname{Re}(z)} \\
& \quad \times\left\{\begin{array}{l}
e^{k|\operatorname{Im}(z)|^{2}+k \operatorname{Re}(z)[1-\operatorname{Re}(z)]+k^{-1} \pi^{2}}, \\
e^{k|\operatorname{Im}(z)|^{2}+k \operatorname{Re}(z)[1-\operatorname{Re}(z)]+(4 k)^{-1} \pi^{2}}, \text { if } T_{1} \geq 0, \text { or } T_{2} \geq 0, \quad z \in \bar{\Sigma}, \\
1, \text { if } T_{j} \geq 0, j=1,2
\end{array}\right.
\end{aligned}
$$

and

$$
\begin{aligned}
& \left\|\overline{T_{2}^{-x} S T_{1}^{-1+x}}\right\|_{\mathcal{B}_{p}\left(\mathcal{H}_{1}, \mathcal{H}_{2}\right)} \leq\left\|S T_{1}^{-1}\right\|_{\mathcal{B}_{p}\left(\mathcal{H}_{1}, \mathcal{H}_{2}\right)}^{1-x}\left\|S^{*} T_{2}^{-1}\right\|_{\mathcal{B}_{p}\left(\mathcal{H}_{2}, \mathcal{H}_{1}\right)}^{x} \\
& \quad \times\left\{\begin{array}{l}
e^{2 \pi[x(1-x)]^{1 / 2}}, \\
e^{\pi[x(1-x)]^{1 / 2}}, \text { if } T_{1} \geq 0, \text { or } T_{2} \geq 0, \quad x \in[0,1] . \\
1, \text { if } T_{j} \geq 0, j=1,2,
\end{array}\right.
\end{aligned}
$$

Proof. One can follow the proof of Corollary 2.10 step by step replacing $\mathcal{B}(\mathcal{H})$ and $\mathcal{B}\left(\mathcal{H}_{j}\right)$ by $\mathcal{B}_{p}(\mathcal{H})$ and $\mathcal{B}_{p}\left(\mathcal{H}_{j}\right), j=1,2$, respectively, applying Theorem 3.2 instead of Theorem 2.8 .

Finally, we recall the following known result in connection with the ideal $\mathcal{B}_{\infty}(\mathcal{H})$ :

Theorem 3.4 ([30, p. 115-116). Suppose that $A(z) \in \mathcal{B}(\mathcal{H}), z \in \bar{\Sigma}$, that $A(\cdot)$ is analytic on $\Sigma$, weakly continuous on $\bar{\Sigma}$. Assume that for some $C_{0}, C_{1} \in(0, \infty)$,

$$
\|A(i y)\|_{\mathcal{B}(\mathcal{H})} \leq C_{0}, \quad\|A(1+i y)\|_{\mathcal{B}(\mathcal{H})} \leq C_{1}, \quad y \in \mathbb{R}
$$


and suppose that for all $f, g \in \mathcal{H}$, there exist $C_{f, g} \in \mathbb{R}$ and $a_{f, g} \in[0, \pi)$, such that

$$
\sup _{z \in \Sigma}\left[e^{-a_{f, g}|\operatorname{Im}(z)|} \ln \left(\left|(f, A(z) g)_{\mathcal{H}}\right|\right)\right] \leq C_{f, g} .
$$

In addition, suppose that

$$
\text { either } \left.A(i y) \in \mathcal{B}_{\infty}(\mathcal{H}), \text { or } A(1+i y) \in \mathcal{B}_{\infty}(\mathcal{H})\right), \quad y \in \mathbb{R} .
$$

Then,

$$
A(z) \in \mathcal{B}_{\infty}(\mathcal{H}), \quad z \in \Sigma .
$$

A condition of the type (3.28) has inadvertently been omitted in [30, p. 115-116].

\section{Extensions to Sectorial Operators}

In this section we revisit Theorems 2.3, 2.8, 3.2, and Corollaries 2.10, 3.3, and replace the self-adjointness hypothesis on $T$ by appropriate sectoriality assumptions.

We start by recalling the definition of a sectorial operator and refer, for instance, to [11, Chs. 2, 3, 7] and [42, Chs. 2, 16] for a detailed treatment.

Definition 4.1. Let $T$ be a densely defined, closed, linear operator in $\mathcal{H}$ and denote by $S_{\omega} \subset \mathbb{C}, \omega \in[0, \pi)$, the open sector

$$
S_{\omega}= \begin{cases}\{z \in \mathbb{C}|z \neq 0,| \arg (z) \mid<\omega\}, & \omega \in(0, \pi), \\ (0, \infty), & \omega=0,\end{cases}
$$

with vertex at $z=0$ along the positive real axis and opening angle $2 \omega$. The operator $T$ is called sectorial of angle $\omega \in[0, \pi)$, denoted by $T \in \operatorname{Sect}(\omega)$, if

$$
\begin{aligned}
& (\alpha) \sigma(T) \subseteq \overline{S_{\omega}}, \\
& (\beta) \text { for all } \omega^{\prime} \in(\omega, \pi), \sup _{z \in \mathbb{C} \backslash \overline{\omega_{\omega^{\prime}}}}\left\|z\left(T-z I_{\mathcal{H}}\right)^{-1}\right\|_{\mathcal{B}(\mathcal{H})}<\infty .
\end{aligned}
$$

One calls

$$
\omega_{T}=\min \{\omega \in[0, \pi] \mid T \in \operatorname{Sect}(\omega)\},
$$

the angle of sectoriality of $T$.

For the remainder of this section we assume that $T$ is sectorial (that is, $T \in$ $\operatorname{Sect}(\omega)$ for some $\omega \in[0, \pi))$ and that $T^{-1} \in \mathcal{B}(\mathcal{H})$.

Then fractional powers $T^{-z}$, with $\operatorname{Re}(z)>0$, of $T$ can be defined by a standard Dunford integral in $\mathcal{B}(\mathcal{H})$ (cf., e.g., [42, Sect. 2.7.1])

$$
T^{-z}=(2 \pi i)^{-1} \oint_{\Gamma} d \zeta \zeta^{-z}\left(T-\zeta I_{\mathcal{H}}\right)^{-1}, \quad \operatorname{Re}(z)>0,
$$

using the principal branch of $\zeta^{-z},\{\zeta \in \mathbb{C}|| \arg (\zeta) \mid<\pi\}$, by excluding the negative real axis, with $\Gamma$ surrounding $\sigma(T)$ clockwise in $(\mathbb{C} \backslash(-\infty, 0]) \cap \rho(T)$ (cf. [42, p. 92] for precise details). An important property of $T^{-z}$ is that

$$
T^{-z} \text { is a } \mathcal{B}(\mathcal{H}) \text {-valued analytic semigroup on }\{z \in \mathbb{C} \mid \operatorname{Re}(z)>0\} \text {. }
$$

Defining imaginary powers of $T$ requires a bit more care. Following [42, p. 105], we introduce the imaginary powers $T^{i s}, s \in \mathbb{R}$, of $T$ as follows:

$$
\begin{aligned}
& T^{i s} f=\underset{z \rightarrow i s, \operatorname{Re}(z)>0}{\operatorname{sim}} T^{-z} f, \\
& f \in \operatorname{dom}\left(T^{i s}\right)=\left\{g \in \mathcal{H} \mid \underset{z \rightarrow i s, \operatorname{Re}(z)>0}{\operatorname{s-lim}} T^{-z} g \text { exists }\right\} .
\end{aligned}
$$


We note that one can define imaginary powers of $T$ also more explicitly as follows: for $s \in \mathbb{R}$, one sets as in [1, p. 153],

$$
T^{i s} f:=\frac{\sin (\pi i s)}{\pi i s} \int_{0}^{\infty} t^{i s}\left(T+t I_{\mathcal{H}}\right)^{-2} T f d t, \quad f \in \operatorname{dom}(T) .
$$

Then the operator $T^{i s}$ is closable for every $s \in \mathbb{R}$ and one defines

$$
T^{i s}:=\overline{\left.T^{i s}\right|_{\operatorname{dom}(T)}}, \quad s \in \mathbb{R} .
$$

We also note that there are several definitions of the fractional (and imaginary) powers in the literature, see, for instance, [11, Section 3.2 and Proposition 3.5.5], [20], [24, Section 4], 25, 38, Section 1], 11, Section 4]. In our setting, all of these definitions coincide (cf. [2]), and we provided the most straightforward one.

To be able to argue as in previous sections one needs to deal with sectorial operators having bounded imaginary powers (BIP).

Definition 4.2. If $T$ is a sectorial operator on $\mathcal{H}$ such that $T^{-1} \in \mathcal{B}(\mathcal{H})$, then $T$ is said to have bounded imaginary powers if $T^{i s} \in \mathcal{B}(\mathcal{H})$ for all $s \in \mathbb{R}$. This is then denoted by $T \in \operatorname{BIP}(\mathcal{H})$.

We recall that if $T$ admits bounded imaginary powers then $\left\{T^{i s}\right\}_{s \in \mathbb{R}}$ is a $C_{0^{-}}$ group on $\mathcal{H}$ (cf. [11, Corollary 3.5.7]). Hence, there exist $\theta \geq 0$ and $N \geq 1$ such that

$$
\left\|T^{i s}\right\|_{\mathcal{B}(\mathcal{H})} \leq N e^{\theta|s|}, \quad s \in \mathbb{R}
$$

and we write $T \in \operatorname{BIP}(N, \theta)$ in this case. Clearly,

$$
\operatorname{BIP}(\mathcal{H})=\bigcup_{N \geqslant 1, \theta \geqslant 0} \operatorname{BIP}(N, \theta) .
$$

We also define the type $\theta_{T}$ of the $C_{0}$-group $\left\{T^{i s}\right\}_{s \in \mathbb{R}}$ by

$$
\theta_{T}:=\inf \left\{\theta \geq 0 \mid \text { there exists } N_{\theta} \geq 1 \text { such that }\left\|T^{i s}\right\|_{\mathcal{B}(\mathcal{H})} \leq N_{\theta} e^{\theta|s|}, s \in \mathbb{R}\right\} \text {. }
$$

The standard example of operators $T$ satisfying $T \in \operatorname{BIP}(\mathcal{H})$ (in addition to the situation described in (2.31) ) are provided by strictly positive self-adjoint operators bounded from below (in this case $T \in \operatorname{BIP}(1,0)$ ) and boundedly invertible, maccretive operators $T$ (in this case $T \in \operatorname{BIP}(1, \pi / 2)$ ). One recalls that $T$ is said to be $m$-accretive (cf. [11, Sect. C.7], [17, [19, Sect. V.3.10], [24, Sect. 4.3], [36, Ch. 2]) if and only if

$$
\overline{\operatorname{dom}(T)}=\mathcal{H}, \quad(-\infty, 0) \subset \rho(T), \quad\left\|\left(T+\lambda I_{\mathcal{H}}\right)^{-1}\right\|_{\mathcal{B}(\mathcal{H})} \leq \lambda^{-1}, \lambda>0 .
$$

The following extension of (4.5) will be vital for the remainder of this section:

Theorem 4.3 (See, e.g., 1, Theorem 4.7.1). If $T \in \operatorname{BIP}(\mathcal{H})$ then $\left\{T^{-z} \mid \operatorname{Re}(z) \geq 0\right\}$ is a strongly continuous semigroup in the closed right half-plane $\{z \in \mathbb{C} \mid \operatorname{Re}(z) \geq 0\}$.

We note that by [11, Proposition 7.0.1] (or [42, p. 101]), $T \in \operatorname{Sect}(\omega)$ if and only if $T^{*} \in \operatorname{Sect}(\omega)$. Moreover, $\left(T^{z}\right)^{*}=\left(T^{*}\right)^{\bar{z}}$ and thus

$$
T \in \operatorname{BIP}(N, \theta) \quad \text { if and only if } T^{*} \in \operatorname{BIP}(N, \theta) .
$$

Theorem 4.3 together with (4.13) permits us to use the three-lines theorem in the present, more general setting of sectorial operators. 
In the special case where $T$ is self-adjoint and strictly positive in $\mathcal{H}$, that is, $T \geq \varepsilon I_{\mathcal{H}}$ for some $\varepsilon>0, T^{\alpha}, \alpha \in \mathbb{C}$, defined on one hand as sectorial operators as above, and on the other by the spectral theorem, coincide (cf., e.g., [24, Sect. 4.3.1], [38, Sect. 1.18.10]). In particular,

$$
\operatorname{dom}\left(T^{\alpha}\right)=\left\{f \in \mathcal{H} \mid\left\|T^{\alpha} f\right\|_{\mathcal{H}}^{2}=\int_{[\varepsilon, \infty]} \lambda^{2 \operatorname{Re}(\alpha)} d\left\|E_{T}(\lambda) f\right\|_{\mathcal{H}}^{2}<\infty\right\}, \quad \alpha \in \mathbb{C},
$$

in this case. Here $\left\{E_{T}(\lambda)\right\}_{\lambda \in \mathbb{R}}$ denotes the family of spectral projections of $T$.

In the remainder of this section, we will use the following set of assumptions:

Hypothesis 4.4. Assume that $T$ is a sectorial operator in $\mathcal{H}$ such that $T^{-1} \in$ $\mathcal{B}(\mathcal{H})$. In addition, we assume that $S$ is a closed operator in $\mathcal{H}$ satisfying

$$
\operatorname{dom}(S) \supseteq \operatorname{dom}(T), \quad \operatorname{dom}\left(S^{*}\right) \supseteq \operatorname{dom}\left(T^{*}\right) .
$$

We start with the analog of Theorem 2.3

Theorem 4.5. Assume Hypothesis 4.4, Then the following facts hold:

(i) The operator $T^{-1} S T$ is well-defined on $\operatorname{dom}\left(T^{2}\right)$, and hence densely defined in $\mathcal{H}$,

(ii) The relation

$$
\operatorname{dom}\left(T^{-1} S T\right) \supseteq \operatorname{dom}\left(T^{2}\right)
$$

$$
\left(T^{-1} S T\right)^{*}=T^{*} S^{*}\left(T^{*}\right)^{-1}
$$

holds, and hence $T^{*} S^{*}\left(T^{*}\right)^{-1}$ is closed in $\mathcal{H}$.

(iii) One infers that

$$
T^{-1} S T \text { is bounded if and only if }\left(T^{-1} S T\right)^{*}=T^{*} S^{*}\left(T^{*}\right)^{-1} \in \mathcal{B}(\mathcal{H}) \text {. }
$$

In case (4.18) holds, then

$$
\overline{T^{-1} S T}=\left(T^{*} S^{*}\left(T^{*}\right)^{-1}\right)^{*}, \quad\left\|\overline{T^{-1} S T}\right\|_{\mathcal{B}(\mathcal{H})}=\left\|T^{*} S^{*}\left(T^{*}\right)^{-1}\right\|_{\mathcal{B}(\mathcal{H})} .
$$

Proof. Since dom $\left(T^{2}\right)$ is an operator core for $T$ (cf. [11, Theorem 3.1.1]), one can follow the proof of Theorem 2.3 line by line. To illustrate this claim we just mention, for instance, the analog of (2.16) which now turns into

$$
\begin{aligned}
\left(\left(T^{-1} S T\right)^{*} f, g\right)_{\mathcal{H}} & =\left(f, T^{-1} S T g\right)_{\mathcal{H}}=\left(\left(T^{*}\right)^{-1} f, S T g\right)_{\mathcal{H}}=\left(S^{*}\left(T^{*}\right)^{-1} f, T g\right)_{\mathcal{H}} \\
& f \in \operatorname{dom}\left(\left(T^{-1} S T\right)^{*}\right), g \in \operatorname{dom}\left(T^{2}\right) \subseteq \operatorname{dom}\left(T^{-1} S T\right),
\end{aligned}
$$

and hence once again extends to all $g \in \operatorname{dom}(T)$ as before in (2.17).

Next, we turn to the analog of Theorem 2.8 and recall the notation used in (4.9):

Theorem 4.6. Assume Hypothesis 4.4. If $T \in \operatorname{BIP}(N, \theta)$, then $T^{-z} S T^{-1+z}, z \in \bar{\Sigma}$, defined on $\operatorname{dom}(T)$, is closable in $\mathcal{H}$, and

$$
\begin{aligned}
\overline{T^{-z} S T^{-1+z}}= & T^{-i \operatorname{Im}(z)}\left[\left|S^{*}\right|^{\operatorname{Re}(z)}\left(T^{*}\right)^{-\operatorname{Re}(z)}\right]^{*} U_{S} \\
& \times|S|^{1-\operatorname{Re}(z)} T^{-1+\operatorname{Re}(z)} T^{i \operatorname{Im}(z)} \in \mathcal{B}(\mathcal{H}), \quad z \in \bar{\Sigma} .
\end{aligned}
$$

In addition, given $k \in(0, \infty)$, one obtains

$$
\begin{aligned}
\left\|\overline{T^{-z} S T^{-1+z}}\right\|_{\mathcal{B}(\mathcal{H})} \leq & N^{2} e^{k(\operatorname{Im}(z))^{2}+k \operatorname{Re}(z)[1-\operatorname{Re}(z)]+k^{-1} \theta^{2}} \\
& \times\left\|S T^{-1}\right\|_{\mathcal{B}(\mathcal{H})}^{1-\operatorname{Re}(z)}\left\|S^{*}\left(T^{*}\right)^{-1}\right\|_{\mathcal{B}(\mathcal{H})}^{\operatorname{Re}(z)}, \quad z \in \bar{\Sigma},
\end{aligned}
$$


and

$$
\left\|\overline{T^{-x} S T^{-1+x}}\right\|_{\mathcal{B}(\mathcal{H})} \leq N^{2} e^{2 \theta[x(1-x)]^{1 / 2}}\left\|S T^{-1}\right\|_{\mathcal{B}(\mathcal{H})}^{1-x}\left\|S^{*}\left(T^{*}\right)^{-1}\right\|_{\mathcal{B}(\mathcal{H})}^{x}, \quad x \in[0,1] .
$$

Proof. Closely examining the first part of the proof of Theorem 2.8 based on Heinz's inequality, one notes that everything up to (2.60) goes through without any change, implying the closability of $T^{-z} S T^{-1+z}$ and the validity of (4.21).

Next, one defines

$$
\begin{array}{r}
\phi_{k}(z)=e^{k z(z-1)}\left(T^{*} f, T^{-1-z} S T^{-2+z} T g\right)_{\mathcal{H}}=e^{k z(z-1)}\left(f, \overline{T^{-z} S T^{-1+z}} g\right)_{\mathcal{H}}, \\
f \in \operatorname{dom}\left(T^{*}\right), g \in \operatorname{dom}(T), z \in \bar{\Sigma}, k \in(0, \infty) .
\end{array}
$$

Then, employing (4.9) and (4.13), one estimates

$$
\begin{aligned}
& \left|\phi_{k}(i y)\right|=e^{-k y^{2}}\left|\left(\left(T^{*}\right)^{i y} f, S T^{-1} T^{i y} g\right)_{\mathcal{H}}\right| \\
& \leq e^{-k y^{2}} N^{2} e^{2 \theta|y|}\left\|S T^{-1}\right\|_{\mathcal{B}(\mathcal{H})}\|f\|_{\mathcal{H}}\|g\|_{\mathcal{H}} \\
& \leq N^{2} e^{k^{-1} \theta^{2}}\left\|S T^{-1}\right\|_{\mathcal{B}(\mathcal{H})}\|f\|_{\mathcal{H}}\|g\|_{\mathcal{H}}, \quad y \in \mathbb{R}, \\
& \left|\phi_{k}(1+i y)\right|=e^{-k y^{2}}\left|\left(\left(T^{*}\right)^{i y} f, \overline{T^{-1} S} T^{i y} g\right)_{\mathcal{H}}\right| \\
& \leq e^{-k y^{2}} N^{2} e^{2 \theta|y|}\left\|\overline{T^{-1} S}\right\|_{\mathcal{B}(\mathcal{H})}\|f\|_{\mathcal{H}}\|g\|_{\mathcal{H}} \\
& =e^{-k y^{2}} N^{2} e^{2 \theta|y|}\left\|S^{*}\left(T^{*}\right)^{-1}\right\|_{\mathcal{B}(\mathcal{H})}\|f\|_{\mathcal{H}}\|g\|_{\mathcal{H}}, \\
& \leq N^{2} e^{k^{-1} \theta^{2}}\left\|S^{*}\left(T^{*}\right)^{-1}\right\|_{\mathcal{B}(\mathcal{H})}\|f\|_{\mathcal{H}}\|g\|_{\mathcal{H}}, \quad y \in \mathbb{R} \\
& \left|\phi_{k}(z)\right|=e^{-k(\operatorname{Im}(z))^{2}+k \operatorname{Re}(z)[\operatorname{Re}(z)-1]}\left|\left(T^{*} f, T^{-1-z} S T^{-2+z} T g\right)_{\mathcal{H}}\right| \\
& \leq e^{-k(\operatorname{Im}(z))^{2}+k \operatorname{Re}(z)[\operatorname{Re}(z)-1]}\left\|T^{-1-\operatorname{Re}(z)-i \operatorname{Im}(z)}\right\|_{\mathcal{B}(\mathcal{H})}\left\|S T^{-1}\right\|_{\mathcal{B}(\mathcal{H})} \\
& \times\left\|T^{-1+\operatorname{Re}(z)+i \operatorname{Im}(z)}\right\|_{\mathcal{B}(\mathcal{H})}\left\|T^{*} f\right\|_{\mathcal{H}}\|T g\|_{\mathcal{H}} \\
& \leq e^{-k(\operatorname{Im}(z))^{2}+k \operatorname{Re}(z)[\operatorname{Re}(z)-1]} N^{2} e^{2 \theta|\operatorname{Im}(z)|}\left\|T^{-1-\operatorname{Re}(z)}\right\|_{\mathcal{B}(\mathcal{H})} \\
& \times\left\|S T^{-1}\right\|_{\mathcal{B}(\mathcal{H})}\left\|T^{-1+\operatorname{Re}(z)}\right\|_{\mathcal{B}(\mathcal{H})}\left\|T^{*} f\right\|_{\mathcal{H}}\|T g\|_{\mathcal{H}} \\
& \leq C_{k}, \quad f \in \operatorname{dom}\left(T^{*}\right), g \in \operatorname{dom}(T), z \in \bar{\Sigma},
\end{aligned}
$$

where $C_{k}=C_{k}(f, g, S, T)>0$ is a finite constant, independent of $z \in \bar{\Sigma}$.

Applying the Hadamard three-lines estimate (2.25) to $\phi(\cdot)$ then yields (4.22) since $\operatorname{dom}(T)$ and $\operatorname{dom}\left(T^{*}\right)$ are dense in $\mathcal{H}$ and $\overline{T^{-z} S T^{-1+z}} \in \mathcal{B}(\mathcal{H}), z \in \bar{\Sigma}$, by (4.21). If $\operatorname{Im}(z)=0$, optimizing (4.22) with respect to $k>0$ implies (4.23).

Remark 4.7. We recall that by McIntosh's theorem (cf. [11, Corollary 4.3.5]), one has

$$
\theta_{T}=\omega_{T}
$$

where $\omega_{T}$ and $\theta_{T}$ are defined by (4.3) and (4.11), respectively. Thus, in principle, one can use $\omega_{T}$ to get estimates cruder than (4.22), (4.23), but then in a priori terms associated with $T$. However, we decided not to pursue this here. The same remark also concerns the statements in the remainder of this section.

In the special case where $T \geq 0$ and $S \in \mathcal{B}(\mathcal{H})$, the estimate (4.23) recovers [35. Lemma 15]. 
Again, these results naturally extend to the situation where $T^{-z} S T^{-1+z}$ is replaced by $T_{2}^{-z} S T_{1}^{-1+z}$ for two sectorial operators $T_{j}$ in $\mathcal{H}_{j}, j=1,2$, having bounded imaginary powers, and once more we now illustrate this in the context of Theorem 4.6 .

Hypothesis 4.8. Assume that $T_{j}$ are sectorial operators in $\mathcal{H}_{j}$ such that $T_{j}^{-1} \in$ $\mathcal{B}\left(\mathcal{H}_{j}\right), j=1,2$. In addition, suppose that $S$ is a closed operator mapping $\operatorname{dom}(S) \subseteq$ $\mathcal{H}_{1}$ into $\mathcal{H}_{2}$, satisfying

$$
\operatorname{dom}(S) \supseteq \operatorname{dom}\left(T_{1}\right) \text { and } \operatorname{dom}\left(S^{*}\right) \supseteq \operatorname{dom}\left(T_{2}^{*}\right) .
$$

Then the analog of Corollary 2.10 reads as follows:

Corollary 4.9. Assume Hypothesis 4.8. If $T_{j} \in \operatorname{BIP}\left(N_{j}, \theta_{j}\right), j=1,2$, then $T_{2}^{-z} S T_{1}^{-1+z}$ defined on $\operatorname{dom}\left(T_{1}\right), z \in \bar{\Sigma}$, is closable, and

$$
\begin{aligned}
\overline{T_{2}^{-z} S T_{1}^{-1+z}}= & T_{2}^{-i \operatorname{Im}(z)}\left[\left|S^{*}\right|^{\operatorname{Re}(z)}\left(T_{2}^{*}\right)^{-\operatorname{Re}(z)}\right]^{*} U_{S} \\
& \times|S|^{1-\operatorname{Re}(z)} T_{1}^{-1+\operatorname{Re}(z)} T_{1}^{i \operatorname{Im}(z)} \in \mathcal{B}\left(\mathcal{H}_{1}, \mathcal{H}_{2}\right), \quad z \in \bar{\Sigma} .
\end{aligned}
$$

In addition, given $k \in(0, \infty)$, one obtains

$$
\begin{aligned}
& \left\|\overline{T_{2}^{-z} S T_{1}^{-1+z}}\right\|_{\mathcal{B}\left(\mathcal{H}_{1}, \mathcal{H}_{2}\right)} \leq N_{1} N_{2} e^{k(\operatorname{Im}(z))^{2}+k \operatorname{Re}(z)[1-\operatorname{Re}(z)]+(4 k)^{-1}\left(\theta_{1}+\theta_{2}\right)^{2}} \\
& \quad \times\left\|S T_{1}^{-1}\right\|_{\mathcal{B}\left(\mathcal{H}_{1}, \mathcal{H}_{2}\right)}^{1-\operatorname{Re}(z)}\left\|S^{*}\left(T_{2}^{*}\right)^{-1}\right\|_{\mathcal{B}\left(\mathcal{H}_{2}, \mathcal{H}_{1}\right)}^{\operatorname{Re}(z)}, z \in \bar{\Sigma}
\end{aligned}
$$

and

$$
\begin{aligned}
& \left\|\overline{T_{2}^{-x} S T_{1}^{-1+x}}\right\|_{\mathcal{B}\left(\mathcal{H}_{1}, \mathcal{H}_{2}\right)} \leq N_{1} N_{2} e^{\left(\theta_{1}+\theta_{2}\right)[x(1-x)]^{1 / 2}} \\
& \quad \times\left\|S T_{1}^{-1}\right\|_{\mathcal{B}\left(\mathcal{H}_{1}, \mathcal{H}_{2}\right)}^{1-x}\left\|S^{*}\left(T_{2}^{*}\right)^{-1}\right\|_{\mathcal{B}\left(\mathcal{H}_{2}, \mathcal{H}_{1}\right)}^{x}, \quad x \in[0,1] .
\end{aligned}
$$

Proof. Again, the $2 \times 2$ block operator formalism introduced in the proof of Corollary 2.10 applies to the case at hand.

We emphasize that (4.31) is not new, it can be found in [42, Lemma 16.3]. Our proof, however, is slightly different.

Finally, we turn to the analogs of Theorem 3.2 and Corollary 3.3.

Theorem 4.10. Assume Hypothesis 4.4. Moreover, let $p \in[1, \infty)$, and suppose that

$$
S T^{-1} \in \mathcal{B}_{p}(\mathcal{H}), \quad S^{*}\left(T^{*}\right)^{-1} \in \mathcal{B}_{p}(\mathcal{H}) .
$$

If $T \in B I P(N, \theta)$, then $T^{-z} S T^{-1+z}, z \in \bar{\Sigma}$, defined on $\operatorname{dom}(T)$, is closable in $\mathcal{H}$, and

$$
\overline{T^{-z} S T^{-1+z}} \in \mathcal{B}_{p}(\mathcal{H}), \quad z \in \bar{\Sigma} .
$$

In addition, given $k \in(0, \infty)$, one obtains

$$
\begin{aligned}
\left\|\overline{T^{-z} S T^{-1+z}}\right\|_{\mathcal{B}_{p}(\mathcal{H})} \leq & N^{2} e^{k(\operatorname{Im}(z))^{2}+k \operatorname{Re}(z)[1-\operatorname{Re}(z)]+k^{-1} \theta^{2}} \\
& \times\left\|S T^{-1}\right\|_{\mathcal{B}_{p}(\mathcal{H})}^{1-\operatorname{Re}(z)}\left\|S^{*}\left(T^{*}\right)^{-1}\right\|_{\mathcal{B}_{p}(\mathcal{H})}^{\operatorname{Re}(z)}, \quad z \in \bar{\Sigma}
\end{aligned}
$$

and

$$
\begin{aligned}
\left\|\overline{T^{-x} S T^{-1+x}}\right\|_{\mathcal{B}_{p}(\mathcal{H})} \leq & N^{2} e^{2 \theta[x(1-x)]^{1 / 2}} \\
& \times\left\|S T^{-1}\right\|_{\mathcal{B}_{p}(\mathcal{H})}^{1-x}\left\|S^{*}\left(T^{*}\right)^{-1}\right\|_{\mathcal{B}_{p}(\mathcal{H})}^{x}, \quad x \in[0,1] .
\end{aligned}
$$


Proof. First we note that Theorem 4.6 applies and hence (4.21)-4.23) are at our disposal. Next, one introduces

$$
A_{k}(z)=e^{k z(z-1)} \overline{T^{-z} S T^{-1+z}}, \quad z \in \bar{\Sigma}, k \in(0, \infty),
$$

and estimates

$$
\begin{aligned}
& \left\|A_{k}(i y)\right\|_{\mathcal{B}_{p}(\mathcal{H})}=e^{-k y^{2}}\left\|T^{-i y} S T^{-1} T^{i y}\right\|_{\mathcal{B}_{p}(\mathcal{H})} \leq e^{-k y^{2}} N^{2} e^{2 \theta|y|}\left\|S T^{-1}\right\|_{\mathcal{B}_{p}(\mathcal{H})} \\
& \leq N^{2} e^{k^{-1} \theta^{2}}\left\|S T^{-1}\right\|_{\mathcal{B}_{p}(\mathcal{H})}, \quad y \in \mathbb{R}, \\
& \left\|A_{k}(1+i y)\right\|_{\mathcal{B}_{p}(\mathcal{H})}=e^{-k y^{2}}\left\|\overline{T^{-1-i y} S T^{i y}}\right\|_{\mathcal{B}_{p}(\mathcal{H})} \\
& =e^{-k y^{2}}\left\|T^{-i y}\left(S^{*}\left(T^{*}\right)^{-1}\right)^{*} T^{i y}\right\|_{\mathcal{B}_{p}(\mathcal{H})} \leq e^{-k y^{2}} N^{2} e^{2 \theta|y|}\left\|S^{*}\left(T^{*}\right)^{-1}\right\|_{\mathcal{B}_{p}(\mathcal{H})} \\
& \leq N^{2} e^{k^{-1} \theta^{2}}\left\|S^{*}\left(T^{*}\right)^{-1}\right\|_{\mathcal{B}_{p}(\mathcal{H})}, \quad y \in \mathbb{R}, \\
& \left\|A_{k}(z)\right\|_{\mathcal{B}(\mathcal{H})}=e^{-k(\operatorname{Im}(z))^{2}+k \operatorname{Re}(z)[\operatorname{Re}(z)-1]} \\
& \times\left\|T^{-i \operatorname{Im}(z)}\left(\left|S^{*}\right|^{\operatorname{Re}(z)}\left(T^{*}\right)^{-\operatorname{Re}(z)}\right)^{*} U_{S}|S|^{1-\operatorname{Re}(z)} T^{-1+\operatorname{Re}(z)} T^{i \operatorname{Im}(z)}\right\|_{\mathcal{B}(\mathcal{H})} \\
& \leq e^{-k(\operatorname{Im}(z))^{2}+k \operatorname{Re}(z)[\operatorname{Re}(z)-1]} N^{2} e^{2 \theta|\operatorname{Im}(z)|}\left\|\left|S^{*}\right|^{\operatorname{Re}(z)}\left(T^{*}\right)^{-\operatorname{Re}(z)}\right\|_{\mathcal{B}(\mathcal{H})} \\
& \times\left\||S|^{1-\operatorname{Re}(z)} T^{-1+\operatorname{Re}(z)}\right\|_{\mathcal{B}(\mathcal{H})} \leq C_{k}, \quad z \in \bar{\Sigma},
\end{aligned}
$$

where $C_{k}=C_{k}(S, T)>0$ is a finite constant, independent of $z \in \bar{\Sigma}$, applying (2.57) and (2.59). Here we used again the generalized polar decomposition (2.51) for $S$ (with $\alpha=\operatorname{Re}(z)$ ).

Applying the Hadamard three-lines estimate (3.5) to $A(\cdot)$ then yields relation (4.34) and the estimate (4.35). If $\operatorname{Im}(z)=0$, optimizing (4.35) with respect to $k>0$ implies (4.36).

Corollary 4.11. In addition to Hypothesis 4.8 , let $p \in[1, \infty)$ and assume that

$$
S T_{1}^{-1} \in \mathcal{B}_{p}\left(\mathcal{H}_{1}, \mathcal{H}_{2}\right), \quad S^{*}\left(T_{2}^{*}\right)^{-1} \in \mathcal{B}_{p}\left(\mathcal{H}_{2}, \mathcal{H}_{1}\right) .
$$

If $T_{j} \in \operatorname{BIP}\left(N_{j}, \theta_{j}\right), j=1,2$, then $T_{2}^{-z} S T_{1}^{-1+z}$ defined on $\operatorname{dom}\left(T_{1}\right), z \in \bar{\Sigma}$, is closable, and

$$
\overline{T_{2}^{-z} S T_{1}^{-1+z}} \in \mathcal{B}_{p}\left(\mathcal{H}_{1}, \mathcal{H}_{2}\right), \quad z \in \bar{\Sigma}
$$

In addition, given $k \in(0, \infty)$, one obtains

$$
\begin{aligned}
& \left\|\overline{T_{2}^{-z} S T_{1}^{-1+z}}\right\|_{\mathcal{B}_{p}\left(\mathcal{H}_{1}, \mathcal{H}_{2}\right)} \leq N_{1} N_{2} e^{k(\operatorname{Im}(z))^{2}+k \operatorname{Re}(z)[1-\operatorname{Re}(z)]+(4 k)^{-1}\left(\theta_{1}+\theta_{2}\right)^{2}} \\
& \quad \times\left\|S T_{1}^{-1}\right\|_{\mathcal{B}_{p}\left(\mathcal{H}_{1}, \mathcal{H}_{2}\right)}^{1-\operatorname{Re}()}\left\|S^{*}\left(T_{2}^{*}\right)^{-1}\right\|_{\mathcal{B}_{p}\left(\mathcal{H}_{2}, \mathcal{H}_{1}\right)}^{\operatorname{Re}(z)}, z \in \bar{\Sigma}
\end{aligned}
$$

and

$$
\begin{aligned}
& \left\|\overline{T_{2}^{-x} S T_{1}^{-1+x}}\right\|_{\mathcal{B}_{p}\left(\mathcal{H}_{1}, \mathcal{H}_{2}\right)} \leq N_{1} N_{2} e^{\left(\theta_{1}+\theta_{2}\right)[x(1-x)]^{1 / 2}} \\
& \quad \times\left\|S T_{1}^{-1}\right\|_{\mathcal{B}_{p}\left(\mathcal{H}_{1}, \mathcal{H}_{2}\right)}^{1-x}\left\|S^{*}\left(T_{2}^{*}\right)^{-1}\right\|_{\mathcal{B}_{p}\left(\mathcal{H}_{2}, \mathcal{H}_{1}\right)}^{x}, \quad x \in[0,1] .
\end{aligned}
$$

Proof. Applying Theorem 4.10, one can follow the proof of Corollary 4.9 (see also Corollary 2.10) step by step replacing $\mathcal{B}(\mathcal{H})$ and $\mathcal{B}\left(\mathcal{H}_{j}\right)$ by $\mathcal{B}_{p}(\mathcal{H})$ and $\mathcal{B}_{p}\left(\mathcal{H}_{j}\right)$, $j=1,2$, respectively.

Acknowledgments. We are indebted to Alexander Gomilko for very helpful discussions. We also thank Matthias Lesch for valuable correspondence. 
F.G. is indebted to all organizers of the Herrnhut Symposium, "Operator Semigroups meet Complex Analysis, Harmonic Analysis and Mathematical Physics" (June 3-7, 2013), and particularly, to Wolfgang Arendt, Ralph Chill, and Yuri Tomilov, for fostering an extraordinarily stimulating atmosphere during the meeting.

\section{REFERENCES}

[1] H. Amann, Linear and Quasilinear Parabolic Problems, Vol. I. Abstract Linear Theory, Monographs in Mathematics, Vol. 89, Birkhäuser, Basel, 1995.

[2] C. J. K. Batty, A. Gomilko, and Yu. Tomilov, Product formulas in functional calculi for sectorial operators, arXiv:1309.0318

[3] C. Bennett and R. Sharpley, Interpolation of Operators, Academic Press, Boston, 1988.

[4] A. Carey, D. Potapov, and F. Sukochev, Spectral flow is the integral of one forms on the Banach manifold of self adjoint Fredholm operators, Adv. Math. 222, 1809-1849 (2009).

[5] T. Furuta, Invitation to Linear Operators. From Matrices to Bounded Linear Operators in a Hilbert Space, Taylor \& Francis, London, 2002.

[6] F. Gesztesy, Y. Latushkin, K. A. Makarov, F. Sukochev, and Y. Tomilov, The index formula and the spectral shift function for relatively trace class perturbations, Adv. Math. 227, 319420 (2011).

[7] F. Gesztesy, M. Malamud, M. Mitrea, and S. Naboko, Generalized polar decompositions for closed operators in Hilbert spaces and some applications, Integral Eq. Operator Theory 64, 83-113 (2009).

[8] I. C. Gohberg and M. G. Krein, Introduction to the Theory of Linear Nonselfadjoint Operators, Translations of Mathematical Monographs, Vol. 18, Amer. Math. Soc., Providence, RI, 1969.

[9] I. C. Gohberg and M. G. Krein, Theory and Applications of Volterra Operators in Hilbert Space, Translations of Mathematical Monographs, Vol. 24, Amer. Math. Soc., Providence, RI, 1970.

[10] L. Grafakos, Classical Fourier Analysis, 2nd ed., Graduate Texts in Mathematics, Vol. 249, Springer, New York, 2008.

[11] M. Haase, The Functional Calculus for Sectorial Operators, Operator Theory: Advances and Applications, Vol. 169, Birkhäuser, Basel, 2006.

[12] E. Heinz, Beiträge zur Störungstheorie der Spektralzerlegung, Math. Ann. 123, 415-438 (1951).

[13] I. I. Hirschman, A convexity theorem for certain groups of transformations, J. Analyse Math. 2, 209-218 (1952).

[14] F. Huang, On the mathematical model for linear elastic systems with analytic damping, SIAM J. Control Optim. 26, 714-724 (1988).

[15] T. Kato, Notes on some inequaliies for linear operators, Math. Ann. 125, 208-212 (1952).

[16] T. Kato, A generalization of the Heinz inequality, Proc. Japan Acad. 37, 305-308 (1961).

[17] T. Kato, Fractional powers of dissipative operators, J. Math. Soc. Japan, 13, 246-274 (1961).

[18] T. Kato, Fractional powers of dissipative operators, II, J. Math. Soc. Japan, 14, 242-248 (1962).

[19] T. Kato, Perturbation Theory for Linear Operators, corr. printing of the 2nd ed., Springer, Berlin, 1980.

[20] M. A. Krasnoselskii, P. P. Zabreiko, E. I. Pustylnik, and P. E. Sobolevskii, Integral Operators in Spaces of Summable Functions, Noordhoff, Leyden, 1976.

[21] S. G. Krein, Ju. I. Petunin, and E. M. Semenov, Interpolation of Linear Operators, Transl. Math. Monographs, Vol. 54, Amer. Math. Soc., Providence, RI, 1982.

[22] M. Lesch, The uniqueness of the spectral flow on spaces of unbounded self-adjoint Fredholm operators, in Spectral Geometry of Manifolds with Boundary and Decomposition of Manifolds, B. Boss-Bavnbek, G. Grubb, and K. P. Wojciechowski (eds.), Contemp. Math. 366, 193-224 (2005).

[23] S. Lord, D. Potapov, and F. Sukochev, Measures from Dixmier traces and zeta functions, J. Funct. Anal. 259, 1915-1949 (2010). 
[24] A. Lunardi, Interpolation Theory, Lecture Notes, Vol. 9, Scuola Normale Superiore Pisa, 2009.

[25] C. Martinez Carracedo and M. Sanz Alix, The Theory of Fractional Powers of Operators, North-Holland Mathematics Studies, Vol. 187, Elsevier, Amsterdam, 2001.

[26] A. McIntosh, Heinz inequalities and perturbation of spectral families, Macquarie Mathematics Reports, Report 79-006, revised, 1980.

[27] D. Potapov and F. Sukochev, Double operator integrals and submajorization, Math. Model. Nat. Phenom. 5, 317-339 (2010).

[28] M. Reed and B. Simon, Methods of Modern Mathematical Physics. II: Fourier Analysis, Self-Adjointness, Academic Press, New York, 1975.

[29] M. Reed and B. Simon, The scattering of classical waves from inhomogeneous media, Math. Z. 155, 163-180 (1977).

[30] M. Reed and B. Simon, Methods of Modern Mathematical Physics. IV: Analysis of Operators, Academic Press, New York, 1978.

[31] W. Rudin, Real and Complex Analysis, 3rd. ed., McGraw-Hill, New York, 1987.

[32] R. Schatten, Norm Ideals of Completely Continuous Operators, Ergebnisse der Mathematik und ihrer Grenzgebiete, Vol. 27, Springer, Berlin, 1960.

[33] B. Simon, Trace Ideals and Their Applications, 2nd ed., Mathematical Surveys and Monographs, Vol. 120, Amer. Math. Soc., Providence, RI, 2005.

[34] E. M. Stein, Interpolation of linear operators, Trans. Amer. Math. Soc. 83, 482-492 (1956).

[35] F. A. Sukochev, On a conjecture of A. Bikchentaev, in "Spectral Analysis, Differential Equations and Mathematical Physics: A Festschrift in Honor of Fritz Gesztesy's 60th Birthday", H. Holden, B. Simon, and G. Teschl (eds.), Proc. Symposia Pure Math., Vol. 87, Amer. Math. Soc., Providence, RI, 2013, pp. 327-339.

[36] H. Tanabe, Equations of Evolution, Monographs and Studies in Mathematics, Vol. 6, Pitman, London, 1979.

[37] K. Tanahashi, A. Uchiyama, and M. Uchiyama, On Schwarz type inequalities, Proc. Amer. Math. Soc. 131, 2549-2552 (2003).

[38] H. Triebel, Interpolation Theory, Function Spaces, Differential Operators, 2nd ed., Barth, Heidelberg, 1995.

[39] M. Uchiyama, Further extension of the Heinz-Kato-Furuta inequality, Proc. Amer. Math. Soc. 127, 2899-2904 (1999).

[40] M. Uchiyama, Operator monotone functions and operator inequalities, Sugaku Expos. 18, 39-52 (2005).

[41] J. Weidmann, Linear Operators in Hilbert Spaces, Graduate Texts in Mathematics, Vol. 68, Springer, New York, 1980.

[42] A. Yagi, Abstract Parabolic Evolution Equations and their Applications, Springer Monographs in Mathematics, Springer, Berlin, 2010.

Department of Mathematics, University of Missouri, Columbia, MO 65211, USA

E-mail address: gesztesyf@missouri.edu

$U R L:$ http://www.math.missouri.edu/personnel/faculty/gesztesyf.html

Department of Mathematics, University of Missouri, Columbia, MO 65211, USA

E-mail address: latushkiny@missouri.edu

$U R L:$ http://www.math.missouri.edu/personnel/faculty/latushkiny.html

School of Mathematics and Statistics, UnSW, Kensington, NSW 2052, Australia

E-mail address: f.sukochev@unsw.edu.au

Faculty of Mathematics and Computer Science, Nicholas Copernicus University, ul. Chopina 12/18, 87-100 Torun, Poland

E-mail address: tomilov@mat.uni.torun.pl 